Article

\title{
Ontogeny of Sex-Related Differences in Foetal Developmental Features, Lipid Availability and Fatty Acid Composition
}

\author{
Consolacion Garcia-Contreras ${ }^{1}$, Marta Vazquez-Gomez ${ }^{2}$, Susana Astiz ${ }^{1}$, Laura Torres-Rovira ${ }^{1}$, \\ Raul Sanchez-Sanchez ${ }^{1}$, Ernesto Gomez-Fidalgo ${ }^{1}$, Jorge Gonzalez ${ }^{3}$, Beatriz Isabel ${ }^{2}$, Ana Rey ${ }^{2}$, \\ Cristina Ovilo ${ }^{1}$ and Antonio Gonzalez-Bulnes ${ }^{1, *}$ \\ 1 Subdirección General de Investigación y Tecnología, Instituto Nacional de Investigación y Tecnología \\ Agraria y Alimentaria, Madrid 28040, Spain; consolacion@inia.es (C.G.-C.); astiz.susana@inia.es (S.A.); \\ torres.laura@inia.es (L.T.-R.); raulss@inia.es (R.S.-S.); fidalgo@inia.es (E.G.-F.); ovilo@inia.es (C.O.) \\ 2 Faculty of Veterinary Sciences, Universidad Complutense de Madrid, Madrid 28040, Spain; \\ mvgomez@ucm.es (M.V.-G.); bisabelr@ucm.es (B.I.); anarey@ucm.es (A.R.) \\ 3 Micros Veterinaria, Leon 24007, Spain; info@microsvet.com \\ * Correspondence: bulnes@inia.es; Tel.: +34-91-347-4022
}

Academic Editor: Marica Bakovic

Received: 13 February 2017; Accepted: 25 May 2017; Published: 31 May 2017

\begin{abstract}
Sex-related differences in lipid availability and fatty acid composition during swine foetal development were investigated. Plasma cholesterol and triglyceride concentrations in the mother were strongly related to the adequacy or inadequacy of foetal development and concomitant activation of protective growth in some organs (brain, heart, liver and spleen). Cholesterol and triglyceride availability was similar in male and female offspring, but female foetuses showed evidence of higher placental transfer of essential fatty acids and synthesis of non-essential fatty acids in muscle and liver. These sex-related differences affected primarily the neutral lipid fraction (triglycerides), which may lead to sex-related postnatal differences in energy partitioning. These results illustrate the strong influence of the maternal lipid profile on foetal development and homeorhesis, and they confirm and extend previous reports that female offspring show better adaptive responses to maternal malnutrition than male offspring. These findings may help guide dietary interventions to ensure adequate fatty acid availability for postnatal development.
\end{abstract}

Keywords: fatty-acids; foetus; lipids; metabolism; nutrition; sex

\section{Introduction}

Prenatal development of humans and animals requires adequate placental supply of oxygen and nutrients [1,2], which, in turn, requires adequate maternal nutrition and placental function. Inadequate placental nutrient supply leads to intrauterine growth restriction (IUGR), resulting in newborns that are small for their gestational age. Such offspring may be predisposed to perinatal morbidity and mortality [3] as well as lifelong chronic non-communicable disorders such as obesity, type II diabetes, hypertension and cardiovascular diseases [4-7].

Among humans, IUGR due to maternal malnutrition shows a much higher incidence in resource-challenged areas $(15 \%)$ than in developed areas $(6 \%)$ [8,9]. In recent years, incidence has been increasing in developed countries because of maternal eating disorders, voluntary intake restriction for aesthetic reasons [10] and abnormal placental development leading to placental insufficiency [11]. This abnormal placental development has been associated with postponement of childbearing, inadequate lifestyle and maternal and gestational factors $[8,12,13]$. 
Previous studies from our group using a swine model of IUGR have highlighted the importance of maternal nutrition for proper foetal development. Maternal malnutrition compromises foetal metabolism and development, causing growth retardation and triggering adaptive changes in the foetus to increase likelihood of survival [14]. Work from other groups has shown that the maternal lipid profile and placental efficiency are strongly related to cholesterol and triglyceride availability for human and other animal foetuses, which affects their viability and growth [15-20]. The developing foetus requires substantial amounts of lipids, mainly polyunsaturated fatty acids (PUFA), the availability of which is determined largely by maternal circulating levels and placental transfer [21]. Impaired placental transfer of PUFA causes or exacerbates IUGR [21-23] and may be caused by lipid peroxidation or other forms of oxidative stress [24,25].

Extensive research in humans and animal models has shown that the sex of the offspring can affect pre- and postnatal development in compromised pregnancies such as IUGR [14,26-31]. Less clear is whether this sex effect extends to lipid homeorhesis. Therefore the present study exploited the power of the pig as a translational model [32] to investigate sex-related differences in lipid availability and fatty acid composition during foetal development. The pig, like humans, is omnivorous and prone to sedentary behaviour and obesity, and the metabolism, cardiovascular system and relative organ sizes in pigs are similar to those in humans [33-36]. A further advantage of studying swine is that insights will impact not only research but also farm productivity. IUGR occurs often in swine because of inadequate maternal nutrition or placental insufficiency [2,37,38], and it limits farm profitability [14].

\section{Results}

Foetuses were obtained from five sows on Gestational Day (GD) $70(n=33)$ or from four sows on GD $90(n=23)$. The average number of foetuses per sow was $6.8 \pm 3.8$ on GD 70 and $5.8 \pm 3.6$ on GD 90. Of the 56 foetuses, 23 were female, of which 13 were recovered on GD 70 and 10 on GD 90; among the 33 male foetuses, 20 were recovered on GD 70 and 13 on GD 90. Hence, the sex ratio was close to 1:1, with males accounting for $57.7 \%$ of GD 70 foetuses and $56.5 \%$ of GD 90 foetuses. Evidence of IUGR was found in eight pregnancies, affecting four GD 70 foetuses (11\%) and four GD 90 foetuses $(17.4 \%)$.

\subsection{Effects of Maternal Metabolic Status on Foetal Development}

The size and weight of normal (non-IUGR) foetuses increased with gestational age (Table 1), as did development of the placenta, liver, kidney and intestine, based on histology (all $p<0.0001$ ).

In these normal foetuses, body development correlated strongly with maternal lipid profile depicted in Figure 1. Specifically, lower maternal plasma cholesterol concentrations correlated with lower foetal body weight $(r=0.777, p<0.05)$ and corpulence in terms of trunk length $(r=0.832)$, thoracic circumference $(r=0.808)$ and abdominal circumference $(r=0.958)($ all $p<0.01)$. Lower maternal plasma cholesterol concentrations also correlated with higher ratios of organ-to-body weight for brain $(r=-0.774)$, heart $(r=-0.752)$, liver $(r=-0.679)$, and spleen $(r=-0.712)($ all $p<0.01)$; these correlations reflect changes to improve foetal viability. Conversely, lower maternal plasma concentrations of high-density lipoprotein cholesterol (HDL-c) correlated with lower ratios of heart-to-body weight $(r=0.677, p<0.05)$. Adaptive foetal growth mechanisms were observed already on GD 70, when lower maternal plasma cholesterol concentrations correlated with higher ratios of head-to-body weight $(r=-0.987, p<0.005)$ and heart-to-body weight $(r=-0.883, p<0.05)$. At the same time, maternal HDL-c concentrations correlated with higher ratios of brain-to-body weight $(r=-0.923)$ and spleen-to-body weight $(r=-0.891$, both $p<0.05)$.

Maternal plasma concentrations of glucose and fructosamine did not affect weight of normal foetuses on GD 70, but they did show an influence on GD 90. Lower plasma fructosamine concentrations correlated with lower foetal body weight $(r=0.964)$ as well as higher ratios of head-to-body weight $(r=-0.962)$ and brain-to-head weight $(r=-0.969$, all $p<0.05)$. Similarly, lower maternal glucose levels correlated with higher ratios of head-to-body weight $(r=-0.959)$ and brain-to-head weight $(r=-0.963$, both $p<0.05)$. 
Table 1. Effect of age and sex on absolute weights and body measurements of male and female foetuses at Gestational Day (GD) 70 and 90 . Mean values for morphometric measurements and absolute body and main organs weights at GD 70 and 90 in female $(\mathrm{F})$ and male $(\mathrm{M})$ foetuses

\begin{tabular}{|c|c|c|c|c|c|c|c|c|c|c|c|}
\hline & & \multirow{2}{*}{\multicolumn{2}{|c|}{70}} & \multirow{2}{*}{\multicolumn{2}{|c|}{90}} & \multirow{3}{*}{ MSE $^{a}$} & \multicolumn{5}{|c|}{$p$-Value } \\
\hline & & & & & & & \multicolumn{2}{|c|}{ Same Age F vs. M } & \multicolumn{2}{|c|}{ Same Sex 70 vs. 90} & \multirow{2}{*}{ Age $\times$ Sex } \\
\hline & & $\mathbf{F}$ & $\mathbf{M}$ & $\mathbf{F}$ & $\mathbf{M}$ & & 70 & 90 & $\mathbf{F}$ & $\mathbf{M}$ & \\
\hline \multirow{6}{*}{$\begin{array}{c}\text { Body } \\
\text { morphometry }\end{array}$} & Body length $(\mathrm{cm})$ & 20.2 & 20.2 & 29.1 & 29.5 & 0.495 & 0.343 & 0.746 & $<0.001$ & $<0.001$ & $<0.001$ \\
\hline & Occipito-nasal length (cm) & 7.7 & 6.6 & 8.4 & 9.2 & 0.403 & 0.045 & 0.193 & 0.241 & $<0.001$ & $<0.001$ \\
\hline & Biparietal diameter (cm) & 3.0 & 2.9 & 3.9 & 4.1 & 0.071 & 0.748 & 0.173 & $<0.001$ & $<0.001$ & $<0.001$ \\
\hline & Trunk diameter $(\mathrm{cm})$ & 2.7 & 2.9 & 4.2 & 4.6 & 0.117 & 0.177 & 0.028 & $<0.001$ & $<0.001$ & $<0.001$ \\
\hline & Trunk circumference $(\mathrm{cm})$ & 11.0 & 11.4 & 16.7 & 17.5 & 0.355 & 0.222 & 0.221 & $<0.001$ & $<0.001$ & $<0.001$ \\
\hline & Abdominal circumference $(\mathrm{cm})$ & 9.3 & 9.8 & 14.5 & 15.3 & 0.439 & 0.294 & 0.277 & $<0.001$ & $<0.001$ & $<0.001$ \\
\hline \multirow{3}{*}{ Body weight } & Body weight (g) & 171.9 & 180.1 & 542.0 & 616.4 & 18.969 & 0.453 & 0.09 & $<0.001$ & $<0.001$ & $<0.001$ \\
\hline & Head weight (g) & 46.8 & 46.6 & 130.4 & 138.9 & 4.086 & 0.948 & 0.340 & $<0.001$ & $<0.001$ & $<0.001$ \\
\hline & Carcass weight (g) & 91.3 & 99.1 & 309.2 & 362.7 & 12.160 & 0.268 & 0.06 & $<0.001$ & $<0.001$ & $<0.001$ \\
\hline \multirow{8}{*}{$\begin{array}{c}\text { Viscerae and } \\
\text { main organs } \\
\text { weight }\end{array}$} & Brain weight (g) & 6.5 & 6.5 & 19.9 & 19.9 & 0.378 & 0.968 & 0.996 & $<0.001$ & $<0.001$ & $<0.001$ \\
\hline & Viscerae weight (g) & 26.3 & 27.7 & 86.5 & 96.5 & 3.245 & 0.495 & 0.168 & $<0.001$ & $<0.001$ & $<0.001$ \\
\hline & Heart weight (g) & 1.4 & 1.5 & 4.7 & 5.2 & 0.193 & 0.616 & 0.233 & $<0.001$ & $<0.001$ & $<0.001$ \\
\hline & Lungs weight (g) & 6.1 & 6.3 & 17.4 & 20.4 & 0.691 & 0.662 & 0.072 & $<0.001$ & $<0.001$ & $<0.001$ \\
\hline & Liver weight (g) & 7.8 & 7.7 & 15.8 & 18.4 & 0.905 & 0.827 & 0.172 & $<0.001$ & $<0.001$ & $<0.001$ \\
\hline & Intestine weight (g) & 5.2 & 4.9 & 21.7 & 25.2 & 1.089 & 0.599 & 0.187 & $<0.001$ & $<0.001$ & $<0.001$ \\
\hline & Spleen weight (g) & 0.2 & 0.2 & 1.2 & 1.5 & 0.053 & 0.425 & 0.018 & $<0.001$ & $<0.001$ & $<0.001$ \\
\hline & Kidneys weight (g) & 2.2 & 2.2 & 5.6 & 6.1 & 0.260 & 0.951 & 0.375 & $<0.001$ & $<0.001$ & $<0.001$ \\
\hline
\end{tabular}

${ }^{\mathrm{a}}$ MSE $=$ Mean square error. 


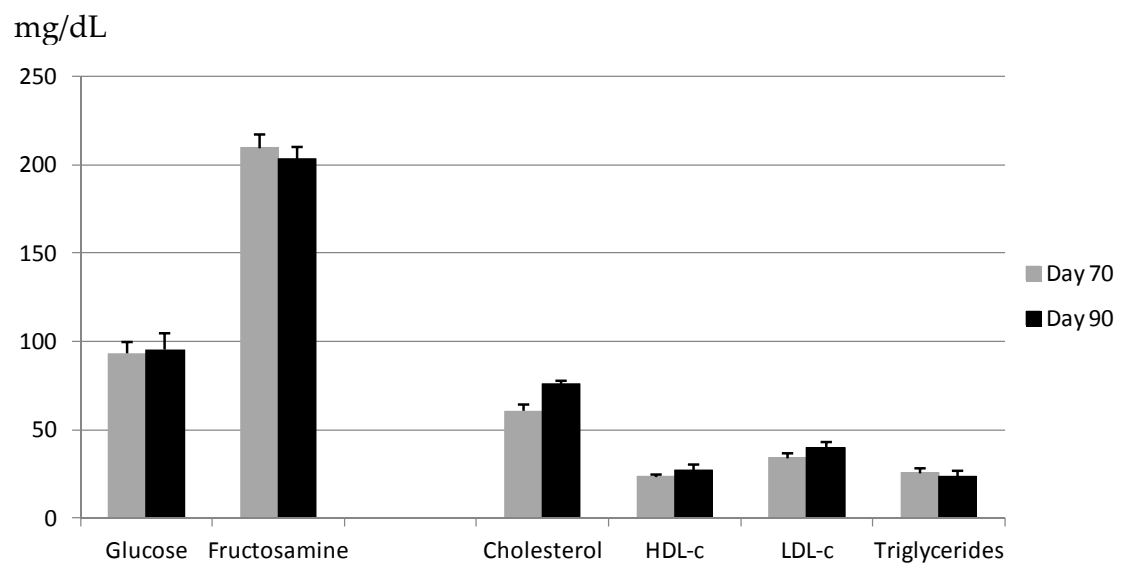

Figure 1. Metabolic profiles of sows, from which foetuses were harvested on Gestational Day (GD) 70 or 90. Mean fasting values (mg/dL) ( \pm SEM) of glucose, fructosamine, total cholesterol, high-density and low-density lipoprotein-cholesterol (HDL-c and LDL-c, respectively) and triglycerides in maternal plasma on the day of harvest.

\subsection{Effects of Foetal Sex on Developmental Trajectories during Pregnancy}

No significant association was observed between offspring sex and foetal weight in normal foetuses (Table 1), although among male foetuses collected on GD 90, we observed a trend toward higher total body weight $(p=0.09)$ and carcass weight $(p=0.06)$ compared to female foetuses. Fat content of the carcass, the longissimus dorsi muscle or the liver did not vary significantly as a function of foetal sex or gestational age (Table 2).

Table 2. Effects of gestational time and sex on foetal adiposity. Mean values (\%) for fat content in the longissimus dorsi muscle (LD) and liver at Gestational Day (GD) 70 and 90 in female (F) and male (M) foetuses.

\begin{tabular}{|c|c|c|c|c|c|c|c|c|c|}
\hline & \multirow{2}{*}{\multicolumn{2}{|c|}{70}} & \multirow{2}{*}{\multicolumn{2}{|c|}{90}} & \multirow{3}{*}{ MSE $^{a}$} & \multicolumn{4}{|c|}{$p$-Value } \\
\hline & & & & & & \multicolumn{2}{|c|}{ Same Age F vs. M } & \multicolumn{2}{|c|}{ Same Sex 70 vs. 90} \\
\hline & $\mathbf{F}$ & $\mathbf{M}$ & $\mathbf{F}$ & $\mathbf{M}$ & & 70 & 90 & $\mathbf{F}$ & $\mathbf{M}$ \\
\hline Fat in LD (\%) & 0.09 & 0.08 & 0.07 & 0.08 & 0.003 & 0.605 & 0.681 & 0.293 & 0.641 \\
\hline Fat in Liver (\%) & 0.14 & 0.14 & 0.14 & 0.14 & 0.002 & 0.434 & 0.522 & 0.814 & 0.294 \\
\hline Fat in carcass & 0.08 & 0.09 & 0.08 & 0.09 & 0.002 & 0.107 & 0.070 & 0.069 & 0.660 \\
\hline
\end{tabular}

Male and female normal foetuses collected on GD 70 did not differ significantly in the relative weights or maturation states of several structures and organs. On GD 90, normal female foetuses showed a significantly higher degree of placental development $(p<0.05)$ and significantly higher ratios of head-to-body weight and brain-to-body weight (both $p<0.05$; Figure 2).

The comparison, on both GD 70 and 90, of foetuses with severe growth restriction and normal foetuses, showed smaller values than their littermates for body weight (GD 70, $126.5 \pm 14.3 \mathrm{~g}$ vs. $183.4 \pm 4.1 \mathrm{~g}, p<0.05$; GD 90, $404.5 \pm 30.9 \mathrm{~g}$ vs. $621.8 \pm 14.5 \mathrm{~g}, p<0.005)$, body length (GD 70, $17.6 \pm 0.9 \mathrm{~cm}$ vs. $20.5 \pm 0.2 \mathrm{~cm}$; GD 90, $27.0 \pm 0.9 \mathrm{~cm}$ vs. $29.8 \pm 0.4 \mathrm{~cm}$; both $p<0.05)$, head weight (GD 70, $35.3 \pm 3.2$ g vs. $48.2 \pm 1 \mathrm{~g}, p<0.05 ; \mathrm{GD} 90,103.5 \pm 6.3 \mathrm{~g}$ vs. $141.9 \pm 3.5 \mathrm{~g}, p<0.005$ ), carcass weight (GD 70, $66.2 \pm 8.9$ g vs. $100 \pm 2.9$ g, $p<0.05 ;$ GD 90, $222.3 \pm 18.7$ g vs. $364.1 \pm 9.6 \mathrm{~g}, p<0.005)$ and total weight of viscerae (GD 70, $18.2 \pm 2$ g vs. $28.3 \pm 0.9 \mathrm{~g}, p<0.01$; GD 90, $64.3 \pm 7.3 \mathrm{~g}$ vs. $98 \pm 2.3 \mathrm{~g}, p<0.05)$. However, the ratio of brain-to-body weight was significantly higher in foetuses with severe growth restriction than in littermates on GD $70(0.048 \pm 0.004$ vs. $0.036 \pm 0.001)$ and GD $90(0.045 \pm 0.004$ vs. $0.033 \pm 0.001$; both $p<0.01)$. This was also observed when only female 
foetuses were examined ( $p<0.0005$ at both ages; Figure 3). On GD 90, the ratios of brain-to-body weight and brain-to-carcass weight were significantly higher in foetuses with severe growth restriction (both $p<0.0005$ ), as were the ratios of liver- and spleen-to-body weight (both $p<0.05$; Figure 3 ).
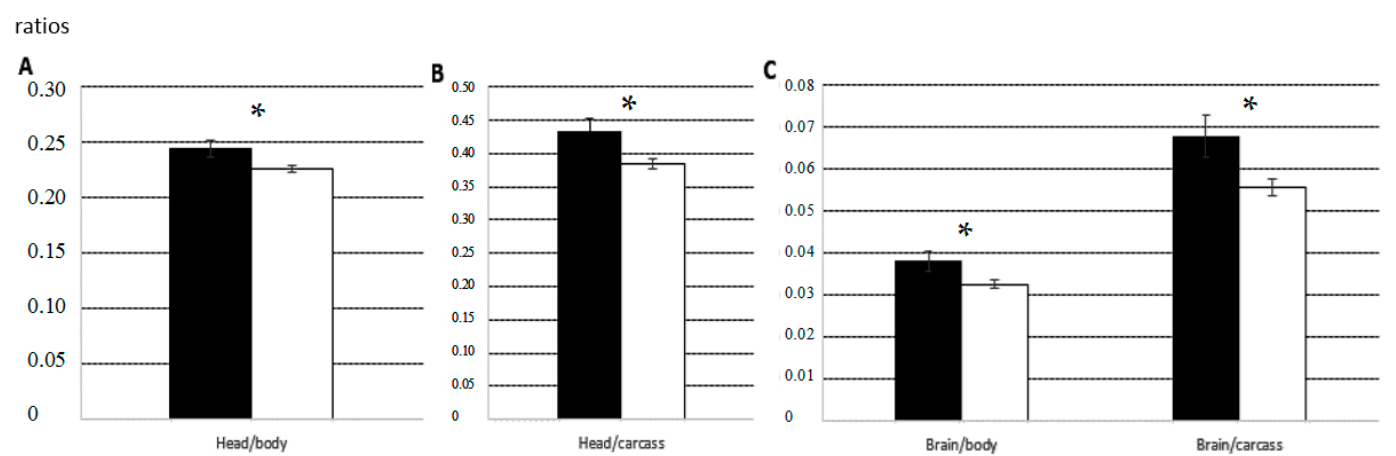

Figure 2. Mean ratios ( \pm SEM) of head-to-body weight (A); head-to-carcass weight (B); and brain-to-body weight and brain-to-carcass weight (C) in female foetuses (black bars) and male foetuses (white bars) on Gestational Day (GD) 90. Asterisks indicate significant differences between males and females. ${ }^{*} p<0.05$.
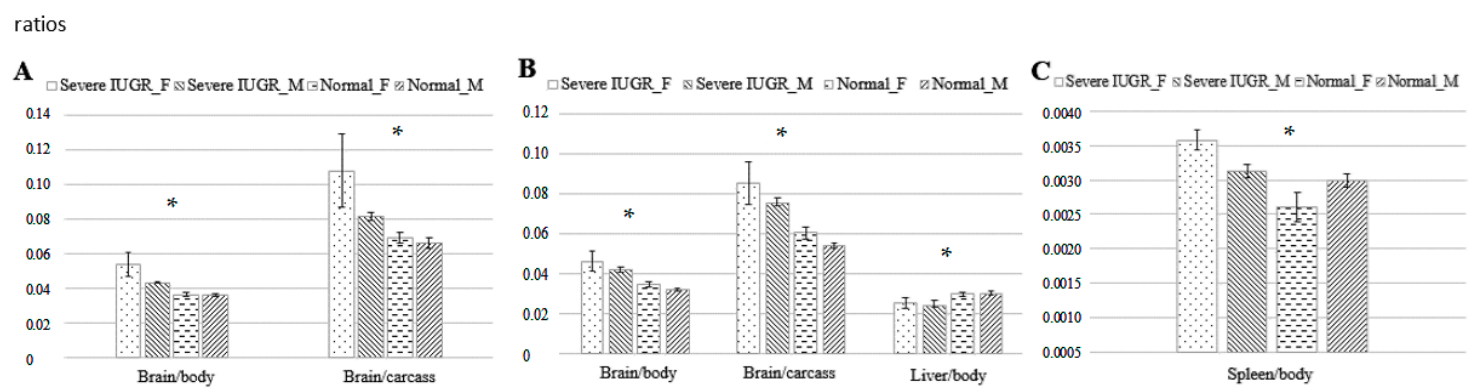

Figure 3. Influence of foetal sex on foetal development under conditions of normal growth or severe IUGR: mean ratios ( \pm mean square error) of brain-to-body weight and brain-to-carcass weight on Gestational Day (GD) 70 (A); ratios of brain-to-body weight, brain-to-carcass weight and liver-to-body weight on GD 90 (B); and ratio of spleen-to-body weight on GD 90 (C). Bars indicate, from left to right, female and male foetuses with severe IUGR, then female and male foetuses showing normal growth. Asterisks indicate the $p$ value associated with differences between the foetal sexes and between foetuses showing IUGR or normal growth. ${ }^{*} p<0.05$.

\subsection{Changes in Foetal Metabolism during Pregnancy and Sex-Related Effects}

Table 3 shows measurements of indicators of glucose and lipid metabolism based on markers in plasma, allantoic and amniotic fluids from normal foetuses. Comparison of measurements on GDs 70 and 90 shows that the availability of foetal triglycerides significantly decreased in foetal blood $(p<0.05)$ and allantoic fluid $(p<0.005)$ during pregnancy. Over the same period, the availability of foetal triglycerides in amniotic fluid increased $(p<0.05)$. Low-density lipoprotein cholesterol (LDL-c) in foetal plasma decreased during pregnancy $(p<0.005)$, while HDL-c increased $(p<0.001)$. Total cholesterol decreased in amniotic fluid $(p<0.0001)$ but increased in allantoic fluid $(p<0.005)$. In both these compartments, HDL-c and LDL-c concentrations remained unchanged during pregnancy. Similar results were obtained for the two foetal sexes, except that LDL-c in blood decreased to a greater extent in females than males $(p<0.05)$. 
Table 3. Effect of age and sex on foetal metabolism. Mean values for parameters of lipids and glucose metabolism at Gestational Day (GD) 70 and 90 in female (F) and male (M) foetuses.

\begin{tabular}{|c|c|c|c|c|c|c|c|c|c|c|c|c|}
\hline & & & \multicolumn{2}{|c|}{70} & \multicolumn{2}{|c|}{90} & \multirow{3}{*}{ MSE $^{a}$} & \multicolumn{5}{|c|}{$p$-Value } \\
\hline & & & \multirow{2}{*}{$\mathbf{F}$} & \multirow{2}{*}{$\mathbf{M}$} & \multirow{2}{*}{$\mathbf{F}$} & \multirow{2}{*}{$\mathbf{M}$} & & \multicolumn{2}{|c|}{ Same Age F vs. $M$} & \multicolumn{2}{|c|}{ Same Sex 70 vs. 90} & \multirow{2}{*}{ Age $\times$ Sex } \\
\hline & & & & & & & & 70 & 90 & $\mathbf{F}$ & $\mathbf{M}$ & \\
\hline \multirow{6}{*}{$\begin{array}{c}\text { Glucose } \\
\text { metabolism }\end{array}$} & \multirow{3}{*}{$\begin{array}{l}\text { Glucose } \\
\text { (mg/dL) }\end{array}$} & Foetal plasma & 24.3 & 19.8 & 64.2 & 58.6 & 5.742 & 0.480 & 0.748 & 0.011 & $<0.001$ & $<0.001$ \\
\hline & & Amniotic fluid & 29.6 & 23.1 & 24.3 & 26.1 & 1.038 & 0.017 & 0.598 & 0.063 & 0.310 & 0.112 \\
\hline & & Allantoic fluid & 7.5 & 5.3 & 21.9 & 22.0 & 1.352 & 0.233 & 0.976 & $<0.001$ & $<0.001$ & $<0.001$ \\
\hline & \multirow{3}{*}{$\begin{array}{l}\text { Fructosamine } \\
\quad(\mathrm{mg} / \mathrm{dL})\end{array}$} & Foetal plasma & 134.8 & 129.6 & 107.8 & 114.9 & 3.147 & 0.583 & 0.190 & 0.003 & 0.094 & 0.012 \\
\hline & & Amniotic fluid & 48.0 & 44.2 & 57.3 & 57.7 & 1.216 & 0.157 & 0.879 & 0.002 & $<0.001$ & $<0.001$ \\
\hline & & Allantoic fluid & 90.2 & 81.6 & 270.7 & 340.8 & 27.390 & 0.592 & 0.568 & 0.016 & 0.001 & $<0.001$ \\
\hline \multirow{12}{*}{$\begin{array}{l}\text { Lipid } \\
\text { metabolism }\end{array}$} & \multirow{3}{*}{$\begin{array}{l}\text { Triglycerides } \\
\text { (mg/dL) }\end{array}$} & Foetal plasma & 39.4 & 32.2 & 28.3 & 28.6 & 1.804 & 0.204 & 0.938 & 0.081 & 0.437 & 0.154 \\
\hline & & Amniotic fluid & 1.8 & 2.5 & 3.6 & 6.3 & 0.701 & 0.070 & 0.435 & 0.048 & 0.114 & 0.113 \\
\hline & & Allantoic fluid & 10.6 & 11.3 & 4.9 & 5.8 & 0.857 & 0.793 & 0.509 & 0.024 & 0.024 & 0.012 \\
\hline & \multirow{3}{*}{$\begin{array}{c}\text { Total } \\
\text { cholesterol } \\
(\mathrm{mg} / \mathrm{dL})\end{array}$} & Foetal plasma & 65.1 & 58.7 & 63.2 & 59.6 & 1.636 & 0.160 & 0.438 & 0.743 & 0.823 & 0.489 \\
\hline & & Amniotic fluid & 4.0 & 3.9 & 2.8 & 2.2 & 0.180 & 0.885 & 0.082 & 0.007 & 0.001 & $<0.001$ \\
\hline & & Allantoic fluid & 2.7 & 2.5 & 4.5 & 6.3 & 0.417 & 0.636 & 0.310 & 0.051 & 0.004 & 0.003 \\
\hline & \multirow{3}{*}{$\begin{array}{l}\text { HDL-c } \\
(\mathrm{mg} / \mathrm{dL})\end{array}$} & Foetal plasma & 12.8 & 13.8 & 21.2 & 18.7 & 0.624 & 0.482 & 0.072 & $<0.001$ & $<0.001$ & $<0.001$ \\
\hline & & Amniotic fluid & 2.2 & 2.5 & 2.1 & 2.4 & 0.068 & 0.189 & 0.116 & 0.529 & 0.507 & 0.191 \\
\hline & & Allantoic fluid & 2.1 & 2.4 & 2.2 & 2.2 & 0.085 & 0.145 & 0.971 & 0.798 & 0.272 & 0.447 \\
\hline & \multirow{3}{*}{$\begin{array}{l}\text { LDL-c } \\
\text { (mg/dL) }\end{array}$} & Foetal plasma & 45.4 & 41.0 & 33.3 & 35.7 & 1.261 & 0.225 & 0.252 & 0.005 & 0.07 & 0.004 \\
\hline & & Amniotic fluid & 3.3 & 3.5 & 3.6 & 3.5 & 0.094 & 0.415 & 0.664 & 0.302 & 0.864 & 0.783 \\
\hline & & Allantoic fluid & 3.1 & 3.4 & 3.2 & 4.3 & 0.233 & 0.257 & 348 & 0.833 & 0.256 & 0.349 \\
\hline
\end{tabular}

${ }^{\mathrm{a}}$ MSE $=$ Square Mean error. 
Similar changes to these described in normal foetuses were observed in the subset of foetuses showing severe IUGR, and no sex effects were observed in this case. Foetuses with severe IUGR showed lower plasma LDL-c concentrations than littermates on GD $90(29.7 \pm 2.5 \mathrm{mg} / \mathrm{dL}$ vs. $35.8 \pm 1.03 \mathrm{mg} / \mathrm{dL}, p<0.05)$.

\subsection{Changes in Foetal Antioxidant/Oxidative Status during Pregnancy and Sex-Related Effects}

Ferric reducing antioxidant power (FRAP), an index of antioxidant capacity, decreased from GD $70(10.8 \pm 1.3 \mu \mathrm{mol} / \mathrm{mL})$ to GD $90(9.9 \pm 1.6 \mu \mathrm{mol} / \mathrm{mL}, p<0.005)$ in samples from normal foetuses, yet the concentration of malondialdehyde (MDA) in plasma, an index of total lipid oxidation, decreased during the same period from $20.6 \pm 0.4 \mathrm{mmol} / \mathrm{mL}$ to $15.8 \pm 0.6 \mathrm{mmol} / \mathrm{mL}(p<0.005)$. Nevertheless, the ratio of MDA to cholesterol, which takes into account lipid availability, indicated less relative oxidation on GD 70 than GD 90 ( $2.8 \pm 0.9$ vs. $3.5 \pm 0.7)$. Similar results were obtained for the ratio of MDA to LDL-c $(4.1 \pm 1.5$ vs. $6.1 \pm 1.2)$ and the ratio of MDA to triglycerides $(5.1 \pm 1.9$ vs. $8.0 \pm 2.8)$ (all $p<0.05 ;$ Figure 4 ).
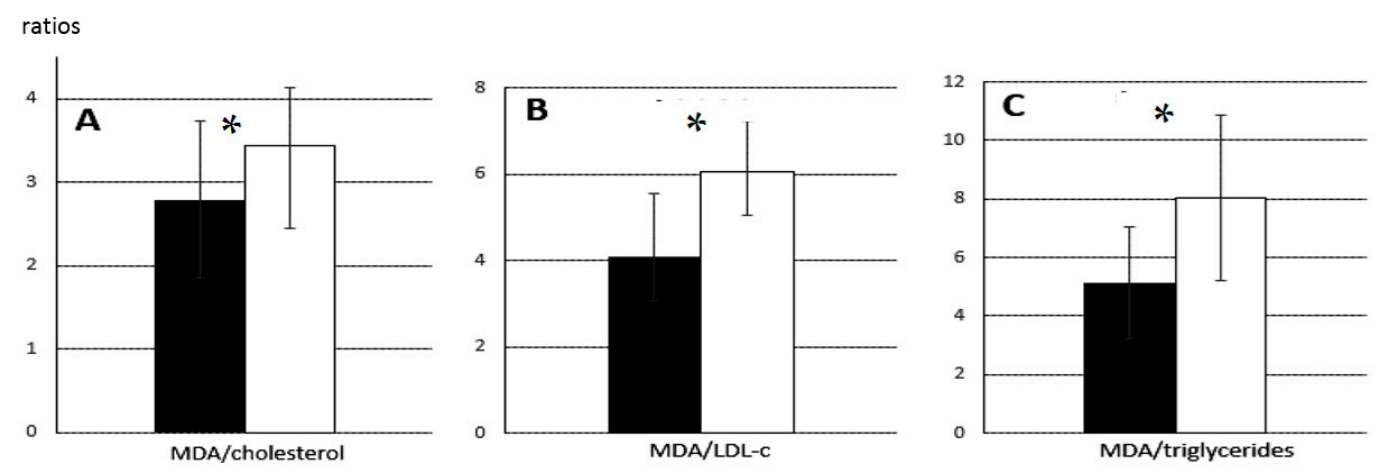

Figure 4. Ratios of MDA to cholesterol, LDL-c and triglycerides of foetuses on Gestational Day (GD) 70 and 90: Mean ratios ( \pm SEM) of: MDA to cholesterol (A); MDA to LDL-c (B); and MDA to triglycerides (C) on GD 70 (black bars) or 90 (white bars). Asterisks indicate significant differences between GD 70 and $90 .{ }^{*} p<0.05$.

\subsection{Changes in Foetal Muscle Fatty Acid Composition during Pregnancy and Sex-Related Effects}

The neutral and polar fatty acid fractions of the longissimus dorsi muscle in normal foetuses varied significantly with gestational age and foetal sex (Tables 4 and 5). On GD 70, the neutral fraction of female normal foetuses contained significantly more $\alpha$-linolenic fatty acid (C18:3n-3) and total saturated fatty acids (SFA) than the neutral fraction of male normal foetuses (both $p<0.05$ ), as well as less cis-vaccenic fatty acid (C18:1n-7) and lower ratios of $\sum \mathrm{n}-6 / \sum \mathrm{n}-3(p<0.01)$ and monounsaturated fatty acids (MUFA) to SFA $(p<0.05)$. All these sex-related differences disappeared by GD 90.

During pregnancy, content of palmitoleic acid (C16:1n-7) and homo- $\gamma$-linolenic acid (C20:3n-6) increased in female normal foetuses (both $p<0.001)$, as did the ratios of $\sum \mathrm{n}-6 / \sum \mathrm{n}-3(p<0.01)$ and C18:1/C18:0 $(p<0.005)$. Similar results were observed for male normal foetuses $\left(\sum \mathrm{n}-6 / \sum \mathrm{n}-3 p<0.001\right.$ and C18:1/C18:0 $p<0.01$, respectively). The content of several fatty acids decreased significantly during pregnancy, including cis-7 hexadecenoic acid (C16:1n-9), heptadecanoic acid (C17:1) and eicosapentaenoic acid (C20:5n-3) (all $p<0.001$ in males and $p<0.05$ in females), as well as stearic acid (C18:0) and erucic acid (C22:1n-9) (all $p<0.05$ in males and females). 
Table 4. Effects of age and sex on foetal neutral fatty-acid composition of longissimus dorsi muscle. Mean values for neutral lipids in the longissimus dorsi muscle at Gestational Day (GD) 70 and 90 in female (F) and male (M) foetuses.

\begin{tabular}{|c|c|c|c|c|c|c|c|c|c|c|}
\hline \multirow[b]{3}{*}{ Fatty Acids (g/100 g Total Fatty Acids) } & \multirow{2}{*}{\multicolumn{2}{|c|}{70}} & \multirow{2}{*}{\multicolumn{2}{|c|}{90}} & \multirow{3}{*}{ MSE $^{a}$} & \multicolumn{5}{|c|}{$p$-Value } \\
\hline & & & & & & \multicolumn{2}{|c|}{ Same Age F vs. $M$} & \multicolumn{2}{|c|}{ Same Sex 70 vs. 90} & \multirow{2}{*}{ Age $\times$ Se $x$} \\
\hline & $\mathbf{F}$ & $\mathbf{M}$ & $\mathbf{F}$ & $\mathbf{M}$ & & 70 & 90 & $\mathbf{F}$ & $\mathbf{M}$ & \\
\hline C14:0 & 3.59 & 3.42 & 3.75 & 3.74 & 0.064 & 0.383 & 0.632 & 0.506 & 0.036 & 0.181 \\
\hline $\mathrm{C} 16: 0$ & 28.97 & 26.79 & 29.33 & 29.25 & 0.375 & 0.069 & 0.549 & 0.809 & 0.001 & 0.021 \\
\hline C16:1n-9 & 4.22 & 4.35 & 3.46 & 3.38 & 0.102 & 0.625 & 0.46 & 0.018 & $<0.001$ & $<0.001$ \\
\hline C16:1n-7 & 3.78 & 4.3 & 7.33 & 7.28 & 0.251 & 0.198 & 0.563 & $<0.001$ & $<0.001$ & $<0.001$ \\
\hline C17:0 & 0.35 & 0.45 & 0.27 & 0.28 & 0.031 & 0.245 & 0.73 & 0.333 & 0.072 & 0.107 \\
\hline C17:1 & 2.96 & 2.82 & 1.68 & 1.77 & 0.089 & 0.364 & 0.177 & $<0.001$ & $<0.001$ & $<0.001$ \\
\hline C18:0 & 13.55 & 13 & 12.21 & 11.55 & 0.218 & 0.351 & 0.375 & 0.035 & 0.012 & 0.006 \\
\hline C18:1n-9 & 21.51 & 21.87 & 22.09 & 22.55 & 0.222 & 0.583 & 0.585 & 0.507 & 0.168 & 0.453 \\
\hline C18:1n-7 & 6.36 & 6.86 & 7.34 & 7.17 & 0.088 & 0.029 & 0.234 & 0.001 & 0.109 & 0.001 \\
\hline C18:2n-6 & 3.34 & 3.19 & 3.64 & 3.96 & 0.079 & 0.363 & 0.163 & 0.151 & $<0.001$ & 0.001 \\
\hline C18:3n-3 & 0.26 & 0.17 & 0.11 & 0.15 & 0.015 & 0.016 & 0.386 & 0.004 & 0.478 & 0.003 \\
\hline C20:3n-9 & 0.57 & 0.63 & 0.56 & 0.54 & 0.010 & 0.063 & 0.147 & 0.757 & $<0.001$ & 0.003 \\
\hline$C 20: 3 n-6$ & 0.24 & 0.26 & 0.43 & 0.47 & 0.016 & 0.569 & 0.098 & $<0.001$ & $<0.001$ & $<0.001$ \\
\hline$C 20: 4 n-6$ & 5.23 & 6.49 & 4.09 & 4.19 & 0.317 & 0.19 & 0.499 & 0.223 & 0.007 & 0.012 \\
\hline C20:5n-3 & 0.29 & 0.28 & 0.2 & 0.19 & 0.008 & 0.718 & 0.605 & $<0.001$ & $<0.001$ & $<0.001$ \\
\hline C22:1n-9 & 0.32 & 0.3 & 0.21 & 0.2 & 0.015 & 0.731 & 0.796 & 0.024 & 0.01 & 0.007 \\
\hline$C 22: 4 n-6$ & 1.44 & 1.68 & 1.05 & 1.11 & 0.078 & 0.297 & 0.219 & 0.125 & 0.003 & 0.006 \\
\hline C22:5n-6 & 0.91 & 1.11 & 0.89 & 0.91 & 0.037 & 0.079 & 0.567 & 0.884 & 0.022 & 0.067 \\
\hline$C 22: 5 n-3$ & 0.37 & 0.35 & 0.3 & 0.3 & 0.017 & 0.701 & 0.621 & 0.171 & 0.276 & 0.373 \\
\hline C22:6n-3 & 1.46 & 1.67 & 1.06 & 1.03 & 0.221 & 0.366 & 0.862 & 0.227 & 0.001 & 0.147 \\
\hline SFA $^{1}$ & 46.45 & 43.66 & 45.55 & 44.81 & 0.407 & 0.029 & 0.345 & 0.566 & 0.166 & 0.063 \\
\hline MUFA $^{2}$ & 39.14 & 40.51 & 42.12 & 42.35 & 0.394 & 0.231 & 0.842 & 0.027 & 0.054 & 0.016 \\
\hline PUFA $^{3}$ & 15.01 & 15.83 & 12.34 & 12.84 & 0.492 & 0.568 & 0.307 & 0.085 & 0.021 & 0.027 \\
\hline $\mathrm{UI}^{4}$ & 0.98 & 1.01 & 0.87 & 0.88 & 0.021 & 0.546 & 0.37 & 0.123 & 0.008 & 0.021 \\
\hline PUFAn-3 ${ }^{5}$ & 3.27 & 2.48 & 1.67 & 1.67 & 0.232 & 0.3 & 0.686 & 0.143 & 0.001 & 0.057 \\
\hline PUFAn- ${ }^{6}$ & 2.59 & 3.05 & 2.37 & 2.49 & 0.108 & 0.173 & 0.249 & 0.558 & 0.037 & 0.084 \\
\hline$\sum n-6 / \sum n-3$ & 0.98 & 1.24 & 1.45 & 1.54 & 0.044 & 0.006 & 0.517 & 0.003 & $<0.001$ & $<0.001$ \\
\hline C18:1/C18:0 & 2.08 & 2.24 & 2.43 & 2.62 & 0.05 & 0.158 & 0.388 & 0.009 & 0.009 & 0.001 \\
\hline MUFA/SFA & 0.85 & 0.93 & 0.93 & 0.95 & 0.013 & 0.046 & 0.618 & 0.135 & 0.488 & 0.052 \\
\hline
\end{tabular}

a MSE = Mean square error. ${ }^{1}$ SFA = Saturated fatty acids; Includes: C14:0, C16:0, C17:0 and C18:0. ${ }^{2}$ MUFA = Monounsaturated fatty acids; Includes: C16:1n-9, C16:1n-7, C17:1, C18:1n-9, C18:1n-7 and C22:1n-9. ${ }^{3}$ PUFA = Polyunsaturated fatty acids: Includes: C18:2n-6, C18:3n-3, C20:3n-9, C20:3n-6, C20:4n-6, C20:5n-3, C22:4n-6, C22:5n-6, C22:5n-3 and C22:6n-3.

${ }^{4} \mathrm{UI}=$ Conversion index. ${ }^{5}$ Includes: C18.2n-6, C20:4n-6 and C22:4n-6. ${ }^{6}$ Includes: C18:3n-3, C20:5n-3 and C22:6n-3. 
Table 5. Effects of age and sex on foetal polar fatty-acid composition of longissimus dorsi muscle. Mean values for polar lipids in the longissimus dorsi muscle at Gestational Day (GD) 70 and 90 in female (F) and male (M) foetuses.

\begin{tabular}{|c|c|c|c|c|c|c|c|c|c|c|}
\hline \multirow[b]{3}{*}{ Fatty Acids (g/100 g Total Fatty Acids) } & \multirow{2}{*}{\multicolumn{2}{|c|}{70}} & \multirow{2}{*}{\multicolumn{2}{|c|}{90}} & \multirow{3}{*}{ MSE $^{a}$} & \multicolumn{5}{|c|}{$p$-Value } \\
\hline & & & & & & \multicolumn{2}{|c|}{ Same Age F vs. $M$} & \multicolumn{2}{|c|}{ Same Sex 70 vs. 90} & \multirow{2}{*}{ Age $\times$ Sex } \\
\hline & F & $\mathbf{M}$ & F & $\mathbf{M}$ & & 70 & 90 & $\mathbf{F}$ & $\mathbf{M}$ & \\
\hline C14:0 & 1.99 & 1.83 & 1.83 & 1.84 & 0.067 & 0.396 & 0.944 & 0.448 & 0.95 & 0.827 \\
\hline C16:0 & 23.69 & 23.68 & 23.79 & 23.15 & 0.103 & 0.967 & 0.082 & 0.747 & 0.062 & 0.113 \\
\hline C16:1n-9 & 2.75 & 2.69 & 2.05 & 1.95 & 0.077 & 0.724 & 0.618 & 0.001 & $<0.001$ & $<0.001$ \\
\hline C16:1n-7 & 2.92 & 3.2 & 3.12 & 3.03 & 0.058 & 0.053 & 0.645 & 0.193 & 0.327 & 0.308 \\
\hline C17:0 & 1.11 & 1.06 & 0.99 & 1.06 & 0.031 & 0.659 & 0.42 & 0.018 & 0.977 & 0.713 \\
\hline C17:1 & 2.34 & 2.34 & 1.45 & 1.55 & 0.061 & 0.944 & 0.088 & $<0.001$ & $<0.001$ & $<0.001$ \\
\hline C18:0 & 11.15 & 11.04 & 11.77 & 11.82 & 0.083 & 0.591 & 0.801 & 0.013 & $<0.001$ & $<0.001$ \\
\hline C18:1n-9 & 21.16 & 21.46 & 21.63 & 21.77 & 0.127 & 0.433 & 0.989 & 0.14 & 0.413 & 0.415 \\
\hline C18:1n-7 & 6.7 & 6.63 & 6.28 & 6.13 & 0.058 & 0.606 & 0.273 & 0.001 & 0.002 & $<0.001$ \\
\hline C18:2n-6 & 2.76 & 2.61 & 4.03 & 4.19 & 0.110 & 0.235 & 0.44 & $<0.001$ & $<0.001$ & $<0.001$ \\
\hline C18:3n-3 & 0.14 & 0.17 & 0.12 & 0.13 & 0.007 & 0.127 & 0.512 & 0.102 & 0.065 & 0.036 \\
\hline C20:1n-9 & 0.7 & 0.76 & 0.84 & 0.85 & 0.016 & 0.229 & 0.804 & 0.004 & 0.024 & 0.003 \\
\hline C20:3n-9 & 0.58 & 0.63 & 0.63 & 0.56 & 0.010 & 0.015 & 0.016 & 0.068 & 0.007 & 0.012 \\
\hline$C 20: 3 n-6$ & 0.23 & 0.25 & 0.57 & 0.67 & 0.030 & 0.382 & 0.14 & $<0.001$ & $<0.001$ & $<0.001$ \\
\hline$C 20: 4 n-6$ & 12.86 & 12.76 & 12.76 & 13.21 & 0.092 & 0.595 & 0.103 & 0.716 & 0.075 & 0.252 \\
\hline C20:5n-3 & 0.31 & 0.31 & 0.3 & 0.27 & 0.008 & 0.974 & 0.252 & 0.757 & 0.055 & 0.181 \\
\hline C22:1n-9 & 0.55 & 0.56 & 0.78 & 0.74 & 0.018 & 0.847 & 0.176 & $<0.001$ & $<0.001$ & $<0.001$ \\
\hline$C 22: 4 n-6$ & 3.02 & 2.97 & 2.01 & 2.11 & 0.070 & 0.541 & 0.074 & $<0.001$ & $<0.001$ & $<0.001$ \\
\hline$C 22: 5 n-6$ & 1.35 & 1.38 & 1.19 & 1.24 & 0.024 & 0.704 & 0.65 & 0.02 & 0.038 & 0.019 \\
\hline$C 22: 5 n-3$ & 0.72 & 0.68 & 0.69 & 0.62 & 0.015 & 0.308 & 0.285 & 0.526 & 0.157 & 0.180 \\
\hline C22:6n-3 & 2.98 & 2.99 & 3.16 & 3.09 & 0.028 & 0.932 & 0.355 & 0.055 & 0.148 & 0.101 \\
\hline SFA $^{1}$ & 37.94 & 37.63 & 38.39 & 37.87 & 0.692 & 0.447 & 0.261 & 0.216 & 0.568 & 0.467 \\
\hline MUFA $^{2}$ & 37.12 & 37.63 & 36.15 & 36.03 & 0.681 & 0.256 & 0.427 & 0.002 & 0.002 & 0.904 \\
\hline PUFA $^{3}$ & 24.95 & 24.74 & 25.47 & 26.09 & 0.477 & 0.401 & 0.215 & 0.219 & 0.002 & 0.195 \\
\hline $\mathrm{UI}^{4}$ & 1.39 & 1.39 & 1.37 & 1.39 & 0.025 & 0.879 & 0.257 & 0.24 & 0.815 & 0.655 \\
\hline PUFAn-3 5 & 4.15 & 4.15 & 4.27 & 4.11 & 0.083 & 0.993 & 0.268 & 0.329 & 0.725 & 0.568 \\
\hline PUFAn-6 ${ }^{6}$ & 20.23 & 19.96 & 20.56 & 21.42 & 0.399 & 0.283 & 0.118 & 0.448 & 0.001 & 0.123 \\
\hline$\sum n-6 / \sum n-3$ & 4.9 & 4.84 & 4.84 & 5.24 & 0.064 & 0.647 & 0.081 & 0.769 & 0.016 & 0.085 \\
\hline C18:1/C18:0 & 0.45 & 0.43 & 0.48 & 0.47 & 0.004 & 0.168 & 0.908 & 0.001 & 0.001 & $<0.001$ \\
\hline MUFA/SFA & 0.98 & 1 & 0.94 & 0.95 & 0.008 & 0.298 & 0.733 & 0.008 & 0.031 & 0.013 \\
\hline
\end{tabular}

a MSE = Mean square error. ${ }^{1}$ SFA = Saturated fatty acids; Includes: C14:0, C16:0, C17:0 and C18:0. ${ }^{2}$ MUFA = Monounsaturated fatty acids; Includes: C16:1n-9, C16:1n-7, C17:1. C18:1n-9, C18:1n-7, C20:1n-9 and C22:1n-9. ${ }^{3}$ PUFA = Polyunsaturated fatty acids: Includes: C18:2n-6, C18:3n-3, C20:3n-9, C20:3n-6, C20:4n-6, C20:5n-3, C22:4n-6, C22:5n-6, C22:5n-3 and C22:6n-3. ${ }^{4} \mathrm{UI}=$ Conversion index. ${ }^{5}$ Includes: C18.2n-6, C20:4n-6 and C22:4n-6. ${ }^{6}$ Includes: C18:3n-3, C20:5n-3 and C22:6n-3. 
During pregnancy, male normal foetuses showed increases in myristic acid (C14:0, $p<0.05)$, palmitic acid (C16:0, $p<0.001)$ and linoleic acid $(\mathrm{C} 18: 2 n-6, p<0.001)$, while female normal foetuses showed increases in cis-vaccenic acid (C18:1n-7, $p<0.01)$ and total MUFA $(p<0.05)$. During pregnancy, male foetuses showed decreases in mead acid (C20:3n-9, $p<0.001)$, adrenic acid (C22:4n-6, $p<0.005)$, arachidonic acid (C20:4n-6, $p<0.01)$, docosapentaenoic acid (C22:5n- $6, p<0.05)$, total PUFA $(p<0.05)$, and the unsaturated index (UI; $p<0.01$ ). Female foetuses showed a significant decrease in $\alpha$-linolenic acid $(p<0.005)$.

Sex-specific differences were also observed in the composition of the polar fatty acid fraction of normal foetuses. Female foetuses had less palmitoleic, $\alpha$-linolenic and mead acids than males (all $p<0.05$ ) on GD 70 and 90. During pregnancy, several fatty acids increased significantly in both sexes, including stearic, $\alpha$-linoleic, homo- $\gamma$-linolenic and erucic acids (all $p<0.0001$ ); docosahexaenoic acid (C22:6n-3, $p<0.05)$; total PUFA $(p<0.05)$; n-6 PUFA $(p<0.05)$; and the C18:1/C18:0 ratio $(p<0.0001)$. Over the same period, several fatty acids decreased significantly in both sexes, including cis-7 hexadecenoic, heptadecanoic, cis-vaccenic, $\alpha$-linolenic, adrenic and docosapentaenoic acids (all $p<0.005)$; and the MUFA/SFA ratio $(p<0.001)$. Female foetuses showed a significant decrease in heptadecanoic acid $(p<0.05)$, while males showed a significant decrease in mead acid $(p<0.05)$ and an increase in $\sum \mathrm{n}-6 / \sum \mathrm{n}-3$ ratio $(p<0.05)$.

\subsection{Changes in Foetal Liver Composition during Pregnancy and Sex-Related Effects}

Like the intramuscular fatty acid composition, the neutral fatty acid composition of the liver of normal foetuses varied significantly with gestational age and foetal sex (Table 6). During pregnancy, several fatty acids increased in both sexes, including myristic, palmitic, palmitoleic, vaccenic and $\alpha$-linoleic acids (all $p<0.0001)$; mead acid $(p<0.005)$; adrenic acid $(p<0.001)$; and the C18:1/C18:0 ratio $(p<0.005)$. Over the same period, several fatty acids decreased in both sexes: margaric (C17:0), heptadecanoic, stearic, and $\alpha$-linolenic acids (all $p<0.0001)$, as well as erucic acid $(p<0.0005)$. In male foetuses, arachidonic and eicosapentaenoic acids decreased (both $p<0.05$ ), while in females, total SFA decreased $(p<0.01)$.

On GD 70 and 90, female foetuses showed higher amounts of stearic acid and total SFA (both $p<0.05)$, but lower amounts of cis-7 hexadecenoic acid $(p<0.005)$, oleic acid $(p<0.05)$, and MUFA $(p<0.01)$, as well as lower ratios of C18:1/C18:0 $(p<0.01)$ and MUFA/SFA $(p<0.005)$. On GD 70 , females showed higher content of heptadecanoic acid $(p<0.05)$ and lower content of cis-vaccenic acid $(p<0.01)$ than males. On GD 90, females showed lower content of heptadecanoic acid $(p<0.05)$ than males.

In contrast, the composition of the polar fatty acid fraction did not vary significantly between male and female normal foetuses (Table 7), with the exception that $\alpha$-linoleic acid content was higher in females than males on GD $70(p<0.05)$. During pregnancy, both sexes showed increases in arachidonic acid $(p<0.0005)$; margaric, stearic, linoleic, and mead acids (all $p<0.0001)$; myristic acid $(p<0.05)$; total PUFA and n-6 PUFA (both $p<0.0001)$; and UI $(p<0.005)$. During the same period, both sexes showed decreases in $\alpha$-linolenic, erucic, cis-7 hexadecenoic and heptadecanoic acids (all $p<0.0001$ ); oleic acid $(p<0.005)$; vaccenic acid $(p<0.05)$; total MUFA $(p<0.0001)$; and the ratios C18:1/C18:0 and MUFA/SFA (both $p<0.0005)$. 
Table 6. Effects of age and sex on foetal neutral fatty-acid composition of liver. Mean values for neutral lipids in the liver at Gestational Day (GD) 70 and 90 in female $(\mathrm{F})$ and male $(\mathrm{M})$ foetuses.

\begin{tabular}{|c|c|c|c|c|c|c|c|c|c|c|}
\hline \multirow[b]{3}{*}{ Fatty Acids (g/100 g Total Fatty Acids) } & \multirow{2}{*}{\multicolumn{2}{|c|}{70}} & \multirow{2}{*}{\multicolumn{2}{|c|}{90}} & \multirow{3}{*}{ SEM $^{a}$} & \multicolumn{5}{|c|}{$p$-Value } \\
\hline & & & & & & \multicolumn{2}{|c|}{ Same Age F vs. $M$} & \multicolumn{2}{|c|}{ Same Sex 70 vs. 90} & \multirow{2}{*}{ Age $\times$ Sex } \\
\hline & F & $\mathbf{M}$ & $\mathbf{F}$ & $\mathbf{M}$ & & 70 & 90 & F & $\mathbf{M}$ & \\
\hline C14:0 & 1.82 & 1.61 & 2.93 & 3.24 & 0.128 & 0.391 & 0.378 & $<0.001$ & $<0.001$ & $<0.001$ \\
\hline C16:0 & 19.02 & 19.67 & 23.14 & 23.25 & 0.336 & 0.345 & 0.945 & $<0.001$ & $<0.001$ & $<0.001$ \\
\hline C16:1n-9 & 1.58 & 2.03 & 1.84 & 2.08 & 0.063 & 0.016 & 0.153 & 0.101 & 0.77 & 0.016 \\
\hline C16:1n-7 & 6.8 & 7.31 & 8.66 & 9.34 & 0.196 & 0.192 & 0.289 & $<0.001$ & $<0.001$ & $<0.001$ \\
\hline C17:0 & 3.5 & 3.06 & 1.6 & 1.59 & 0.125 & 0.028 & 0.887 & $<0.001$ & $<0.001$ & $<0.001$ \\
\hline C17:1 & 2.19 & 2.29 & 1.22 & 1.36 & 0.072 & 0.392 & 0.049 & $<0.001$ & $<0.001$ & $<0.001$ \\
\hline C18:0 & 22.89 & 18.94 & 16.22 & 14.77 & 0.656 & 0.031 & 0.191 & 0.001 & 0.008 & $<0.001$ \\
\hline C18:1n-9 & 18.94 & 19.8 & 17.67 & 20.68 & 0.376 & 0.365 & 0.04 & 0.249 & 0.383 & 0.059 \\
\hline C18:1n-7 & 5.6 & 6.42 & 7.16 & 6.87 & 0.125 & 0.011 & 0.26 & $<0.001$ & 0.166 & $<0.001$ \\
\hline C18:2n-6 & 2.29 & 2.39 & 3.24 & 3.14 & 0.085 & 0.533 & 0.92 & $<0.001$ & $<0.001$ & $<0.001$ \\
\hline C18:3n-3 & 0.37 & 0.36 & 0.26 & 0.26 & 0.010 & 0.783 & 0.808 & $<0.001$ & $<0.001$ & $<0.001$ \\
\hline C20:1n-9 & 0.26 & 0.41 & 0.24 & 0.25 & 0.045 & 0.361 & 0.998 & 0.6 & 0.319 & 0.451 \\
\hline C20:3n-9 & 0.29 & 0.29 & 0.37 & 0.32 & 0.009 & 0.841 & 0.216 & 0.003 & 0.126 & 0.004 \\
\hline C20:4n-6 & 12.16 & 12.99 & 12.98 & 10.61 & 0.373 & 0.344 & 0.169 & 0.488 & 0.018 & 0.084 \\
\hline$C 20: 5 n-3$ & 0.1 & 0.11 & 0.08 & 0.26 & 0.033 & 0.811 & 0.3 & 0.411 & 0.172 & 0.221 \\
\hline C22:1n-9 & 0.47 & 0.37 & 0.28 & 0.24 & 0.022 & 0.087 & 0.689 & 0.007 & 0.011 & 0.001 \\
\hline$C 22: 4 n-6$ & 0.43 & 0.43 & 0.66 & 0.54 & 0.023 & 0.938 & 0.169 & 0.011 & 0.012 & 0.001 \\
\hline C22:5n-3 & 0.84 & 1.12 & 0.95 & 0.78 & 0.049 & 0.069 & 0.17 & 0.334 & 0.025 & 0.038 \\
\hline$C 22: 6 n-3$ & 0.43 & 0.4 & 0.51 & 0.4 & 0.018 & 0.519 & 0.194 & 0.152 & 0.918 & 0.164 \\
\hline SFA $^{1}$ & 47.23 & 43.28 & 43.89 & 42.85 & 0.467 & 0.009 & 0.029 & 0.009 & 0.726 & 0.002 \\
\hline MUFA $^{2}$ & 35.84 & 38.63 & 37.07 & 40.83 & 0.564 & 0.04 & 0.107 & 0.445 & 0.138 & 0.014 \\
\hline PUFA $^{3}$ & 16.93 & 18.09 & 19.04 & 16.32 & 0.469 & 0.314 & 0.215 & 0.157 & 0.164 & 0.234 \\
\hline $\mathrm{UI}^{4}$ & 1 & 1.08 & 1.08 & 1.01 & 0.016 & 0.087 & 0.253 & 0.093 & 0.128 & 0.145 \\
\hline SUMN3 ${ }^{5}$ & 1.75 & 1.99 & 1.8 & 1.7 & 0.063 & 0.155 & 0.793 & 0.697 & 0.138 & 0.267 \\
\hline SUMN6 ${ }^{6}$ & 14.89 & 15.81 & 16.88 & 14.3 & 0.422 & 0.362 & 0.204 & 0.151 & 0.179 & 0.220 \\
\hline N6/N3 & 8.58 & 8.23 & 9.41 & 8.77 & 0.199 & 0.463 & 0.453 & 0.108 & 0.357 & 0.225 \\
\hline SUMC18:1/C18:0 & 1.12 & 1.47 & 1.57 & 1.93 & 0.063 & 0.011 & 0.103 & 0.003 & 0.006 & $<0.001$ \\
\hline MUFA/SFA & 0.77 & 0.91 & 0.85 & 0.95 & 0.019 & 0.007 & 0.067 & 0.096 & 0.321 & 0.002 \\
\hline
\end{tabular}

a MSE = Mean square error. ${ }^{1}$ SFA = Saturated fatty acids; Includes: C14:0, C16:0, C17:0 and C18:0. ${ }^{2}$ MUFA = Monounsaturated fatty acids; Includes: C16:1n-9, C16:1n-7, C17:1. C18:1n-9, C18:1n-7 and C22:1n-9. ${ }^{3}$ PUFA = Polyunsaturated fatty acids: Includes: C18:2n-6, C18:3n-3, C20:3n-9, C20:3n-6, C20:4n-6, C20:5n-3, C22:4n-6 C22:5n-6, C22:5n-3 and C22:6n-3. ${ }^{4} \mathrm{UI}=$ Conversion index. ${ }^{5}$ Includes: C18.2n-6, C20:4n-6 and C22:4n-6. ${ }^{6}$ Includes: C18:3n-3, C20:5n-3 and C22:6n-3. 
Table 7. Effects of age and sex on foetal polar fatty-acid composition of liver. Mean values (\%) for polar lipids in the liver at Gestational Day (GD) 70 and 90 in female (F) and male (M) foetuses.

\begin{tabular}{|c|c|c|c|c|c|c|c|c|c|c|}
\hline \multirow[b]{3}{*}{ Fatty Acids (g/100 g Total Fatty Acids) } & \multirow{2}{*}{\multicolumn{2}{|c|}{70}} & \multirow{2}{*}{\multicolumn{2}{|c|}{90}} & \multirow{3}{*}{ MSE $^{a}$} & \multicolumn{5}{|c|}{$p$-Value } \\
\hline & & & & & & \multicolumn{2}{|c|}{ Same Age F vs. $M$} & \multicolumn{2}{|c|}{ Same Sex 70 vs. 90} & \multirow{2}{*}{ Age $\times$ Sex } \\
\hline & F & $\mathbf{M}$ & F & $\mathbf{M}$ & & 70 & 90 & F & M & \\
\hline C14:0 & 1.73 & 1.66 & 1.89 & 1.8 & 0.033 & 0.45 & 0.524 & 0.168 & 0.084 & 0.088 \\
\hline C16:0 & 22.83 & 21.93 & 23.13 & 22.39 & 0.217 & 0.184 & 0.188 & 0.689 & 0.389 & 0.215 \\
\hline C16:1n-9 & 1.77 & 1.73 & 1.34 & 1.39 & 0.038 & 0.638 & 0.197 & 0.001 & $<0.001$ & $<0.001$ \\
\hline C16:1n-7 & 5.11 & 4.84 & 5.11 & 4.96 & 0.064 & 0.162 & 0.561 & 0.999 & 0.502 & 0.351 \\
\hline C17:0 & 3.09 & 3.05 & 1.72 & 1.75 & 0.100 & 0.764 & 0.797 & $<0.001$ & $<0.001$ & $<0.001$ \\
\hline C17:1 & 1.7 & 1.7 & 0.86 & 0.83 & 0.064 & 0.988 & 0.435 & $<0.001$ & $<0.001$ & $<0.001$ \\
\hline C18:0 & 18 & 18.08 & 19.17 & 19.93 & 0.165 & 0.819 & 0.165 & 0.014 & $<0.001$ & $<0.001$ \\
\hline C18:1n-9 & 14.09 & 13.7 & 12.08 & 12.18 & 0.274 & 0.583 & 0.963 & 0.021 & 0.032 & 0.016 \\
\hline C18:1n-7 & 8.03 & 8.19 & 7.28 & 7.13 & 0.204 & 0.784 & 0.727 & 0.176 & 0.077 & 0.158 \\
\hline C18:2n-6 & 2.38 & 2.15 & 2.82 & 2.68 & 0.055 & 0.033 & 0.549 & 0.005 & 0.001 & $<0.001$ \\
\hline C18:3n-3 & 0.32 & 0.32 & 0.2 & 0.2 & 0.014 & 0.97 & 0.652 & 0.004 & 0.001 & $<0.001$ \\
\hline C20:1n-9 & 0.17 & 0.17 & 0.18 & 0.18 & 0.004 & 0.978 & 0.266 & 0.388 & 0.842 & 0.824 \\
\hline C20:3n-9 & 0.3 & 0.3 & 0.39 & 0.4 & 0.010 & 0.768 & 0.751 & 0.01 & $<0.001$ & $<0.001$ \\
\hline C20:4n-6 & 17.07 & 18.57 & 19.88 & 20.36 & 0.300 & 0.076 & 0.231 & 0.004 & 0.009 & $<0.001$ \\
\hline$C 20: 5 n-3$ & 0.15 & 0.13 & 0.23 & 0.21 & 0.012 & 0.625 & 0.622 & 0.032 & 0.011 & 0.007 \\
\hline C22:1n-9 & 0.4 & 0.43 & 0.29 & 0.3 & 0.015 & 0.549 & 0.591 & 0.003 & 0.002 & $<0.001$ \\
\hline$C 22: 4 n-6$ & 0.56 & 0.53 & 0.92 & 0.83 & 0.041 & 0.674 & 0.543 & 0.016 & 0.001 & 0.001 \\
\hline C22:5n-3 & 1.57 & 1.8 & 1.68 & 1.73 & 0.069 & 0.262 & 0.918 & 0.557 & 0.74 & 0.678 \\
\hline$C 22: 6 n-3$ & 0.72 & 0.71 & 0.84 & 0.76 & 0.023 & 0.907 & 0.23 & 0.145 & 0.429 & 0.279 \\
\hline SFA $^{1}$ & 45.66 & 44.72 & 45.92 & 45.87 & 0.305 & 0.321 & 0.873 & 0.797 & 0.157 & 0.405 \\
\hline MUFA $^{2}$ & 31.27 & 30.76 & 27.13 & 26.97 & 0.461 & 0.64 & 0.822 & 0.002 & 0.002 & $<0.001$ \\
\hline PUFA $^{3}$ & 23.07 & 24.51 & 26.95 & 27.16 & 0.376 & 0.148 & 0.576 & 0.001 & 0.003 & $<0.001$ \\
\hline $\mathrm{UI}^{4}$ & 1.21 & 1.27 & 1.32 & 1.33 & 0.012 & 0.108 & 0.462 & 0.007 & 0.047 & 0.002 \\
\hline SUMN3 ${ }^{5}$ & 2.76 & 2.96 & 2.95 & 2.9 & 0.072 & 0.366 & 0.779 & 0.373 & 0.772 & 0.752 \\
\hline SUMN6 ${ }^{6}$ & 20.01 & 21.25 & 23.61 & 23.86 & 0.332 & 0.134 & 0.492 & 0.001 & 0.001 & $<0.001$ \\
\hline N6/N3 & 7.4 & 7.62 & 8.19 & 8.3 & 0.221 & 0.749 & 0.714 & 0.126 & 0.33 & 0.446 \\
\hline SUMC18:1/C18:0 & 1.23 & 1.22 & 1.02 & 0.98 & 0.033 & 0.85 & 0.623 & 0.026 & 0.005 & 0.004 \\
\hline MUFA/SFA & 0.69 & 0.69 & 0.59 & 0.59 & 0.014 & 0.922 & 0.895 & 0.019 & 0.007 & 0.515 \\
\hline
\end{tabular}

a MSE = Mean square error. ${ }^{1}$ SFA = Saturated fatty acids; Includes: C14:0, C16:0, C17:0 and C18:0. ${ }^{2}$ MUFA = Monounsaturated fatty acids; Includes: C16:1n-9, C16:1n-7, C17:1. C18:1n-9, C18:1n-7 and C22:1n-9. ${ }^{3}$ PUFA = Polyunsaturated fatty acids: Includes: C18:2n-6, C18:3n-3, C20:3n-9, C20:3n-6, C20:4n-6, C20:5n-3, C22:4n-6, C22:5n-6, C22:5n-3 and C22:6n-3. ${ }^{4} \mathrm{UI}=$ Conversion index. ${ }^{5}$ Includes: C18.2n-6, C20:4n-6 and C22:4n-6. ${ }^{6}$ Includes: C18:3n-3, C20:5n-3 and C22:6n-3. 


\section{Discussion}

The results of the present study support the prominent role of the maternal metabolic profile on foetal development and homeorhesis and provide new insights on the effects of offspring sex on fetoplacental development, lipid availability and fatty acid composition at non-adipose tissues involved in metabolism regulation (muscle and liver). These data may be considered relevant for humans since despite differences in placentation, human and pig foetuses and pregnant females show similar lipid metabolism and distribution [39].

In the current study, lower cholesterol concentration in maternal plasma correlated with deficiencies in foetal development, which it is consistently with previous studies on GD 42 [16]. In contrast, poor availability of glucose, the main energy source for developing foetuses [40], affected foetal development on GD 90 but not on GD 70 in our study, consistent with reports that hypoglycaemia in early development is non-critical and can be compensated [41].

The higher growth of the brain when compared to total body observed in the foetuses affected by IUGR is consistent with the "brain-sparing effect" firstly described by Rudolph (1984) [42]. There was also a higher growth of other organs (mainly heart, liver and spleen), which is also consistent with studies in humans documenting "heart- and liver-sparing" cardiovascular adaptations-analogous to the brain-sparing effect-in response to foetal malnutrition and hypoxia [43,44]. Previous studies address that changes in the growth, morphology and function of various organs are dependant on the timing and severity of nutritional restriction [45,46], with specifically a determinant effect triggered by lipid availability. At the same time, our results support previous work indicating sex-specific differences in the growth of the different organs [27,31].

Maternal undernutrition in our study clearly affected foetal lipid availability. The developing foetus and placenta require large amounts of lipids for: (a) synthesis of cholesterol, which acts as a key constituent of cell membranes and organelles and is the precursor of a range of hormones and metabolic regulators necessary for successful pregnancy [47-50]; (b) secretion of key products such as lipoproteins; and (c) storage of triglycerides [47-49]. Although placental and foetal tissues can synthesize lipids de novo [47,51], the building blocks must be taken up by the placenta, i.e. maternal free fatty acids; triglycerides, which placental lipases hydrolyse into fatty acid constituents; and lipoprotein-associated cholesterol $[19,21,23,51]$. Triglycerides are a major source of energy for the foetus [52,53], but a balance is needed: low levels of triglycerides delay growth, while high levels can cause foetal macrosomia [54]. Availability of lipids to the fetoplacental unit depends on de novo synthesis by the foetus as well as maternal transfer. Cholesterol, for example, can reach the foetal circulatory system after crossing the syncytiotrophoblast as LDL-c [49,55]. In pig foetuses, fatty acid availability and composition depend more on foetal synthesis from precursors transferred from the mother than on direct maternal transfer [56-61], since fatty acids do not easily cross the placenta in ruminants, pigs or horses [62].

Lipid availability to the foetoplacental unit also depends on the antioxidant/oxidative status of the foetus. Our results suggest that, during pregnancy, antioxidant capacity decreases and lipid peroxidation increases in foetuses affected by maternal undernutrition, as suggested by previous studies [24-26]. These changes may explain the lower lipid availability later in pregnancy.

Cholesterol and triglyceride availability in our study did not differ significantly between male and female foetuses, consistently with prior studies [56]. Our data further show that the two sexes showed similar antioxidant capacity and lipid peroxidation. In other words, foetal development was affected to a much greater extent by gestational age—and therefore by nutritional restriction — than by sex. Nevertheless, we did observe sex-related differences in how the fatty acid composition of non-adipose tissues involved in metabolism regulation (muscle and liver) changed between GD 70 and 90 in foetuses affected by maternal undernutrition.

Our data indicate significant sex-related differences in content of essential fatty acids, which are so-called because they must be obtained from maternal transfer in the case of foetuses $[22,63]$ or from diet in the case of adults [64]. The major essential fatty acids are linolenic PUFA (an omega-3 fatty 
acid) and linoleic PUFA (an omega-6 fatty acid); the long-chain omega-3 PUFA eicosapentaenoic and docosahexaenoic acids and the long-chain omega-6 PUFA gamma-linolenic and arachidonic acids are also essential. Future work should examine the maternal and/or placental factors that may drive the sex-dependent differences in essential fatty acid availability that we observed.

Most of the differences in fatty acid composition between male and female foetuses that we observed in muscle and liver belonged to the neutral fraction corresponding to triglycerides, which are an essential energy source [19]. Smaller differences were observed in the polar fraction corresponding to phospholipids, which constitute cell membranes and are essential for tissue development [19], so sex-related differences in fatty acid composition are most related to energy partitioning than to organ development.

We found that male foetuses had a higher $n-6 / n-3$ ratio than females, and a high n- $6 / n-3$ ratio appears to be deleterious [65], corresponding to the prodromal phase of insulin resistance [66]. Optimal development depends on adequate availability of n-6 [67], while n-3 may improve insulin function. Our results are consistent with previous reports that alterations in lipid metabolism and insulin regulation appear early in the development of male foetuses under limited nutrition [68-71]. Indeed, males in our study showed a significantly higher ratio of MUFA to SFA in muscle triglycerides than females on GD 70, although this difference was no longer significant on GD 90. On GD 70 and 90, males showed lower stearic acid content in the liver, and this acid is considered to protect metabolic health [72,73]. Males also showed higher ratios of MUFA to SFA and C18:1 to C18:0, indicating higher stearoyl-CoA desaturase activity. Although we observed few sex-related differences in the changes in phospholipid composition of muscle or liver during pregnancy, we did observe that females had higher content of linoleic acid in the liver than males on GD 70, and that males had higher content of mead acid in the muscle on GD 70 and 90 . The greater content of mead acid in males likely reflects a worse homeorhesis state, since synthesis of mead or eicosatrienoic acid occurs in response to severe deficiency of fatty acids, mainly linoleic acid [74,75].

\section{Material and Methods}

\subsection{Ethics Statement}

These experiments were performed according to the Spanish Policy for Animal Protection RD53/2013, which complies with the European Union Directive 2010/63/UE on the protection of animals used for research. The experimental procedures were specifically assessed and approved by the INIA Committee of Ethics in Animal Research (report CEEA 2013/036, finally approved on 19 February 2014). Sows were housed in INIA animal facilities, which meet local, national and European requirements for Scientific Procedure Establishments.

\subsection{Animals and Experimental Procedures}

The study involved a total of 56 foetuses obtained from 9 multiparous purebred Iberian sows with an average body weight of $147.7 \pm 16.0 \mathrm{~kg}$, which became pregnant after cycle synchronization with altrenogest (Regumate ${ }^{\circledR}$, MSD, Boxmeer, The Netherlands) and insemination with cooled semen from a purebred Iberian boar. All sows and the boar were homozygous for the LEPRC.1987T allele based on pyrosequencing [76].

Sows were fed with a standard grain-based food diet with the following mean component values: dry matter, $89.8 \%$; crude protein, $15.1 \%$; fat, $2.8 \%$; and metabolizable energy, $3.0 \mathrm{Mcal} / \mathrm{kg}$. Diet analysis (Table 8 ) showed that the most abundant fatty acids (FA) were palmitic $(18.7 \%)$, oleic $(23.2 \%)$ and linoleic acids (46.5\%). 
Table 8. Fatty acid composition (g/100 g total fatty acids) of the experimental diet.

\begin{tabular}{cc}
\hline Fatty Acids & $\mathbf{\%}$ \\
\hline C14:0 & 0.488 \\
C16:0 & 18.722 \\
C16:1n-9 & 0.173 \\
C16:1n7 & 0.578 \\
C17:0 & 0.540 \\
C17:1 & 0.126 \\
C18:0 & 3.706 \\
C18:1n-9 & 23.201 \\
C18:1n-7 & 0.906 \\
C18:2n-6 & 46.492 \\
C18:3n-3 & 3.355 \\
C20:1n-9 & 0.648 \\
C20:4n-6 & 0.000 \\
C20:5n-3 & 0.123 \\
C22:1n-9 & 0.268 \\
C22:5n-3 & 0.297 \\
C22:6n-3 & 0.377 \\
\hline
\end{tabular}

The amount of food was adjusted to fulfil individual daily maintenance requirements, based on data from the British Society of Animal Science, from the start of the experimental period until GD 35 [77]. On this day, all sows were weighed and the amount of food offered to each sow was adjusted to fulfil $50 \%$ of their daily maintenance requirements, which has been shown to increase IUGR incidence [78]. Every day, a single food ration was weighed out and given to each sow in her individual pen; hence, the diet of each female was adjusted to her own weight.

Foetuses were obtained on GD 70 from five sows or on GD 90 from four sows. These gestational time points correspond to approximately $60 \%$ and $80 \%$ of a 112 -day gestation typical for this breed, corresponding to 24 and 32 weeks of human pregnancy, respectively. These time points were chosen because until GD 70 of swine pregnancy, lipid anabolism and foetal metabolism resemble maternal metabolism [79]; around GD 90, foetal metabolism is independently regulated, and foetal development is more affected by nutrient availability [80].

On GD 70 and 90, blood samples were drawn from the orbital sinus of sows that had fasted for approximately $16 \mathrm{~h}$. Samples were collected in sterile, heparinised 4-mL vacuum tubes (Vacutainer ${ }^{\mathrm{TM}}$ Systems Europe, Meylan, France) and were immediately centrifuged at $1500 \mathrm{~g}$ for $15 \mathrm{~min}$. The plasma was separated and biobanked into polypropylene vials at $-80^{\circ} \mathrm{C}$ until they were assayed for metabolic biomarkers including glycaemic values and lipid profile.

\subsection{Sampling of Placentas and Foetuses}

Animals were euthanised by stunning and exsanguination, in compliance with RD53/2013 standard procedures, and the entire genital tracts were immediately collected for morphometric evaluation and foetal sampling. The contents of the uterus were exposed, and conceptus position was recorded. In each normal non-IUGR foetus, the allantoic and amniotic fluids were obtained by aspiration through the chorioallantoic and amniochorionic membranes, and blood samples were drawn from the heart and/or umbilical cord using heparinised syringes. Blood was processed as described above for sows, while allantoic and amniotic fluids were centrifuged at $1500 \times g$ for $15 \mathrm{~min}$ and supernatants were biobanked into polypropylene vials at $-80{ }^{\circ} \mathrm{C}$ until they were assayed for metabolic biomarkers (including glycaemic values and lipid profile) and antioxidant/oxidative status. Rectangular sections of the uterine wall were collected, fixed in $4 \%$ paraformaldehyde, embedded in paraffin and stained using hematoxylin-eosin. Individual sections of the placentas were measured morphometrically as described [81] to obtain the average width of the placental folds, which served as an index of placental maturation. 


\subsection{Evaluation of Foetal Sex and Morphological Features}

Foetal sex was determined by visual inspection immediately after recovery; detailed pregnancy-by-pregnancy information is shown in Table 9. Body length (crown-rump length), head size (occipito-nasal length and biparietal diameter) and corpulence (thoracic and abdominal circumferences) were measured in all the normal and IUGR foetuses. Total foetus weight was determined, then the head was separated from the trunk at the atlanto-occipital union, and head and trunk were weighed separately. All viscerae were obtained and weighed together immediately, and then the brain, heart, lungs, liver, intestine, kidneys, spleen and pancreas were weighed separately. Ratios of head-to-body weight, brain-to-head weight, and the weight of total viscera and individual organs (brain, heart, lungs, liver, kidneys, intestine, pancreas, and spleen) relative to body weight were calculated.

Table 9. Detailed information (total number and percentage) on sex distribution (total, male and female foetuses) in the pregnancies studied in the current trial.

\begin{tabular}{ccccccc}
\hline \multirow{2}{*}{ Sow ID } & GD & \multicolumn{5}{c}{ Distribution of Foetuses } \\
\cline { 3 - 7 } & & T (n) & F (n) & F (\%) & M (n) & M (\%) \\
\hline 1331 & 70 & 5 & 2 & 40.0 & 3 & 60.0 \\
1335 & 70 & 13 & 5 & 38.5 & 8 & 61.5 \\
1346 & 70 & 7 & 3 & 42.9 & 4 & 57.1 \\
1347 & 70 & 3 & 1 & 33.3 & 2 & 66.7 \\
1418 & 70 & 5 & 3 & 60.0 & 2 & 40.0 \\
1339 & 90 & 4 & 1 & 25.0 & 3 & 75.0 \\
1402 & 90 & 11 & 5 & 45.5 & 6 & 54.5 \\
1411 & 90 & 5 & 2 & 40.0 & 3 & 60.0 \\
1416 & 90 & 3 & 2 & 66.7 & 1 & 33.3 \\
\hline
\end{tabular}

Samples from liver, kidneys, duodenum and ileum were fixed in $4 \%$ paraformaldehyde, embedded in paraffin and stained with hematoxylin-eosin. The degree of maturation of these organs was assessed by evaluating the number of glomeruli with a well-defined glomerular tuft in the kidney [82], the decrease of hepatic haematopoiesis in the liver [83] and villus height and crypt depth in the intestine [84]. This assessment was made by an investigator blinded to sow and foetus details.

\subsection{Evaluation of Maternal and Foetal Metabolic Status}

Lipid profile parameters (triglycerides, total cholesterol, HDL-c, LDL-c) were measured in maternal plasma as well as in plasma, allantoic and amniotic fluids of normal foetuses. Assays were performed using a clinical chemistry analyser (Saturno 300 plus, Crony Instruments s.r.l., Rome, Italy), according to the manufacturer's instructions.

\subsection{Evaluation of Foetal Adiposity and Fat Composition}

Total fat was quantified in carcasses, and the total fat percentage and fatty acid composition (in $\mathrm{g} / 100$ ) were determined in intramuscular fat and liver of normal foetuses. For this purpose, samples from longissimus dorsi muscle and the left liver lobe were biobanked at $-80{ }^{\circ} \mathrm{C}$ until they were assayed as described [85]. Intramuscular fat and liver fat were extracted from $300 \mathrm{mg}$ of lyophilised and homogenised samples using the Ball-mill procedure [86]. Fatty acids in the total lipid extracts were identified and quantified by gas chromatography (HP6890, Hewlett Packard, Avondale, PA, USA) after methylation, as described in $[87,88]$. Fatty acid methyl esters were fractionated on a cross-linked polyethylene glycol capillary column $(30 \mathrm{~cm} \times 0.32 \mathrm{~mm} \times 0.25 \mu \mathrm{m}$, Hewlett Packard Innowax $)$ and a temperature gradient from $170{ }^{\circ} \mathrm{C}$ to $245^{\circ} \mathrm{C}$. The injector and detector were maintained at $250{ }^{\circ} \mathrm{C}$. Neutral lipid fractions (triglycerides) and polar lipid fractions (phospholipids) were analysed using gas chromatography after passing them through aminopropyl minicolumns previously activated with $7.5 \mathrm{~mL}$ of hexane as described [89]. The percentages of individual fatty acids were used to 
calculate proportions of SFA, MUFA and PUFA, as well as total n-3 and n- 6 and their ratio $\left(\sum n-6 / \sum n-3\right)$. The unsaturation index (UI) was obtained from the ratio of MUFA to SFA, and the activity of the stearoyl-CoA desaturase enzyme 1 was inferred from the ratio of the enzyme product, oleic acid (C18:1n-9), to the enzyme substrate, stearic acid (C18:0).

\subsection{Evaluation of Foetal Antioxidant/Lipid Oxidative Status}

In normal foetuses, values for total antioxidant capacity were assayed using the ferric reducing antioxidant power assay (FRAP) as previously described [90], while lipid peroxidation was assessed by measuring MDA $(\mathrm{mmol} / \mathrm{mL})$ using the thiobarbituric acid reaction and HPLC separation with fluorescence detection as previously described [91].

\subsection{Statistical Analyses}

Data were analysed using SPSS 22.0 (IBM Corp., Armonk, NY, USA). Based on previous studies [42], foetuses with severe growth restriction were defined as those with a body weight more than one standard deviation below the litter mean value. Among the 33 foetuses collected on GD 70, four $(11 \%)$ were classified as showing severe growth restriction. Among the 23 foetuses collected on GD 90, four (17.4\%) were so classified. Effects of gestational age (GD 70 vs. 90), sex (female vs. male) and growth restriction on developmental traits, adiposity, fatty-acid composition, and metabolic and foetal oxidative status were assessed by two-way ANOVA. Duncan's post-hoc test was performed to check differences among groups in multiple comparisons. Relationships between maternal metabolic biomarkers and features of foetuses showing normal growth were explored using Pearson correlation. The sow was considered the experimental unit for all variables in order to avoid biasing the results according to litter size: foetuses with the same sex and development (normal or IUGR) from the same sow were averaged together, giving one data point per sow. All results were expressed as mean \pm SEM and statistical significance was accepted from $p<0.05$.

\section{Conclusions}

The present study supports the importance of the maternal lipid profile and placental transfer of lipids, mainly essential fatty acids, for foetal development, and it confirms and extends previous studies suggesting that female foetuses show better adaptive responses in the form of greater synthesis of non-essential fatty acids and better transfer of essential fatty acids, resulting in better metabolic indexes. These sex-related differences were observed primarily in the neutral lipid fraction (triglycerides), suggesting a strong influence of sex on postnatal energy partitioning. These results may help guide future studies on understanding and optimising the maternal diet and placenta transfer capacity in order to meet foetal requirements for fatty acids and prevent the postnatal problems associated with insufficient prenatal fatty acid availability.

Acknowledgments: The authors thank the INIA animal staff for assistance with animal care and P. Cuesta and I. Cano (Department of Research Support, Universidad Complutense de Madrid) for statistical analyses. This work was supported by funds from the Ministry of Economy and Competitiveness (project AGL2013-48121-C3-0-R), co-funded by FEDER. CGC, MVG, SA and AGB are members of the EU COST-Action BM1308 “Sharing Advances on Large Animal Models (SALAAM)".

Author Contributions: Consolacion Garcia-Contreras, Susana Astiz, Beatriz Isabel, Cristina Ovilo and Antonio Gonzalez-Bulnes conceived and designed the experiments; Consolacion Garcia-Contreras, Marta Vazquez-Gomez, Susana Astiz, Laura Torres-Rovira, Ernesto Gomez-Fidalgo, Raul Sanchez-Sanchez, Jorge Gonzalez, Beatriz Isabel, Ana Rey, Cristina Ovilo and Antonio Gonzalez-Bulnes performed the experiments; Consolacion Garcia-Contreras, Marta Vazquez-Gomez, Susana Astiz, Beatriz Isabel, Ana Rey and Antonio Gonzalez-Bulnes analysed the data; Consolacion Garcia-Contreras, Susana Astiz and Antonio Gonzalez-Bulnes wrote the paper; and Marta Vazquez-Gomez, Laura Torres-Rovira, Ernesto Gomez-Fidalgo, Raul Sanchez-Sanchez, Jorge Gonzalez, Beatriz Isabel, Ana Rey and Cristina Ovilo revised the paper.

Conflicts of Interest: The authors declare no conflicts of interest. The funding sponsors had no role in the design of the study; in the collection, analyses, or interpretation of data; in the writing of the manuscript; or in the decision to publish the results. 


\section{References}

1. Vuguin, P.M. Animal models for small for gestational age and fetal programming of adult disease. Horm. Res. 2007, 68, 113-123. [PubMed]

2. Wu, G.; Bazer, F.W.; Wallace, J.M.; Spencer, T.E. Board-invited review: Intrauterine growth retardation: Implications for the animal sciences. J. Anim. Sci. 2006, 84, 2316-2337. [CrossRef] [PubMed]

3. Bhutta, Z.A.; Das, J.K.; Rizvi, A.; Gaffey, M.F.; Walker, N.; Horton, S.; Webb, P.; Lartey, A.; Black, R.E. Lancet Nutrition Interventions Review Group; Maternal and Child Nutrition Study Group. Evidence-based interventions for improvement of maternal and child nutrition: What can be done and at what cost? Lancet 2013, 382, 452-477. [CrossRef]

4. Hovi, P.; Andersson, S.; Eriksson, J.G.; Jarvenpaa, A.L.; Strang-Karlsson, S.; Mäkitie, O.; Kajantie, E. Glucose regulation in young adults with very low birth weight. N. Engl. J. Med. 2007, 356, 2053-2063. [CrossRef] [PubMed]

5. Ibañez, L.; Lopez-Bermejo, A.; Suarez, L.; Marcos, M.V.; Diaz, M.; de Zegher, F. Visceral adiposity without overweight in children born small for gestational age. J. Clin. Endocrinol. Metab. 2008, 93, 2079-2083. [CrossRef] [PubMed]

6. Ross, M.G.; Desai, M. Developmental programming of offspring obesity, adipogenesis, and appetite. Clin. Obstet. Gynecol. 2013, 56, 529-536. [CrossRef] [PubMed]

7. Tam, W.H.; Ma, R.C.; Yang, X.; Li, A.M.; Ko, G.T.; Kong, A.P.; Lao, T.T.; Chan, M.H.; Lam, C.W.; Chan, J.C. Glucose intolerance and cardiometabolic risk in adolescents exposed to maternal gestational diabetes: A 15-year follow-up study. Diabetes Care 2010, 33, 1382-1384. [CrossRef] [PubMed]

8. Baschat, A.A. Fetal responses to placental insufficiency: An update. BJOG 2004, 111, 1031-1041. [CrossRef] [PubMed]

9. Ergaz, Z.; Avgil, M.; Ornoy, A. Intrauterine growth restriction-etiology and consequences: What do we know about the human situation and experimental animal models? Reprod. Toxicol. 2005, 20, 301-322. [CrossRef] [PubMed]

10. Brett, K.E.; Ferraro, Z.M.; Yockell-Lelievre, J.; Gruslin, A.; Adamo, K.B. Maternal-fetal nutrient transport in pregnancy pathologies: The role of the placenta. Int. J. Mol. Sci. 2014, 15, 16153-16185. [CrossRef] [PubMed]

11. Ghidini, A. Idiopathic fetal growth restriction: A pathophysiologic approach. Obstet. Gynecol. Surv. 1996, 51, 376-382. [CrossRef] [PubMed]

12. Patterson, A.J.; Zhang, L. Hypoxia and fetal heart development. Curr. Mol. Med. 2010, 10, 653-666. [CrossRef] [PubMed]

13. Teramo, K.A. Obstetric problems in diabetic pregnancy-The role of fetal hypoxia. Best Pract. Res. Clin. Endocrinol. Metab. 2010, 24, 663-671. [CrossRef] [PubMed]

14. Gonzalez-Bulnes, A.; Astiz, S.; Ovilo, C.; Lopez-Bote, C.J.; Torres-Rovira, L.; Barbero, A.; Ayuso, M.; Garcia-Contreras, C.; Vazquez-Gomez, M. Developmental Origins of Health and Disease in swine: Implications for animal production and biomedical research. Theriogenology 2016, 86, 110-119. [CrossRef] [PubMed]

15. Gonzalez-Bulnes, A.; Ovilo, C. Genetic basis, nutritional challenges and adaptive responses in the prenatal origin of obesity and type-2 diabetes. Curr. Diabetes Rev. 2012, 8, 144-154. [CrossRef] [PubMed]

16. Gonzalez-Bulnes, A.; Torres-Rovira, L.; Ovilo, C.; Astiz, S.; Gomez-Izquierdo, E.; Gonzalez-Añover, P.; Pallares, P.; Perez-Solana, M.L.; Sanchez-Sanchez, R. Reproductive; endocrine and metabolic feto-maternal features and placental gene expression in a swine breed with obesity/leptin resistance. Gen. Comp. Endocrinol. 2012, 176, 94-101. [CrossRef] [PubMed]

17. Herrera, E. Lipid metabolism in pregnancy and its consequences in the fetus and newborn. Endocrine 2002, 19, 43-55. [CrossRef]

18. Herrera, E. Implications of dietary fatty acids during pregnancy on placental; fetal and postnatal development-a review. Placenta 2002, 23 (Suppl. SA), S9-S19. [CrossRef] [PubMed]

19. Herrera, E.; Amusquivar, E.; Lopez-Soldado, I.; Ortega, H. Maternal lipid metabolism and placental lipid transfer. Horm. Res. 2006, 65 (Suppl. S3), 59-64. [CrossRef] [PubMed]

20. Parlakgumus, H.A.; Aytac, P.C.; Kalayci, H.; Tarim, E. First trimester maternal lipid levels and serum markers of small- and large-for-gestational age infants. J. Matern. Fetal. Neonatal. Med. 2014, 27, 48-51. [CrossRef] [PubMed] 
21. Jones, M.L.; Mark, P.J.; Waddell, B.J. Maternal dietary omega-3 fatty acids and placental function. Reproduction 2014, 147, R143-R152. [CrossRef] [PubMed]

22. Bobiński, R.; Mikulska, M. The ins and outs of maternal-fetal fatty acid metabolism. Acta Biochimica Polonica 2015, 62, 499-507. [CrossRef] [PubMed]

23. Herrera, E.; Ortega-Senovilla, H. Lipid metabolism during pregnancy and its implications for fetal growth. Curr. Pharm. Biotechnol. 2014, 15, 24-31. [CrossRef] [PubMed]

24. Biri, A.; Bozkurt, N.; Turp, A.; Kavutcu, M.; Himmetoglu, O. Role of oxidative stress in intrauterine growth restriction. Gynecol. Obstet. Investig. 2007, 64, 187-192. [CrossRef] [PubMed]

25. Kamath, U.; Rao, G.; Kamath, S.U.; Rai, L. Maternal and fetal indicators of oxidative stress during intrauterine growth retardation (IUGR). Indian J. Clin. Biochem. 2006, 21, 111-115. [CrossRef] [PubMed]

26. Gupta, P.; Narang, M.; Banerjee, B.D.; Basu, S. Oxidative stress in term small for gestational age neonates born to undernourished mothers: A case control study. BMC Pediatr. 2004, 4, 14. [CrossRef] [PubMed]

27. Aiken, C.E.; Ozanne, S.E. Sex differences in developmental programming models. Reproduction 2013, 145, R1-R13. [CrossRef] [PubMed]

28. Clifton, V.L.; Murphy, V.E. Maternal asthma as a model for examining fetal sex-specific effects on maternal physiology and placental mechanisms that regulate human fetal growth. Placenta 2004, 25 (Suppl. SA), S45-S52. [CrossRef] [PubMed]

29. Cuffe, J.S.; Dickinson, H.; Simmons, D.G.; Moritz, K.M. Sex specific changes in placental growth and MAPK following short term maternal dexamethasone exposure in the mouse. Placenta 2011, 32, 981-989. [CrossRef] [PubMed]

30. Cuffe, J.S.; O'Sullivan, L.; Simmons, D.G.; Anderson, S.T.; Moritz, K.M. Maternal corticosterone exposure in the mouse has sex-specific effects on placental growth and mRNA expression. Endocrinology 2012, 153, 5500-5511. [CrossRef] [PubMed]

31. Moritz, K.M.; Cuffe, J.S.; Wilson, L.B.; Dickinson, H.; Wlodek, M.E.; Simmons, D.G.; Denton, K.M. Review: Sex specific programming: A critical role for the renal renin-angiotensin system. Placenta 2010, 31, S40-S46. [CrossRef] [PubMed]

32. Gonzalez-Bulnes, A; Astiz, S.; Vazquez-Gomez, M.; Garcia-Contreras, C. Developmental origins of metabolic disorders: The need for biomarker candidates and therapeutic targets from adequate preclinical models. EuPA Open Proteom. 2016, 10, 50-55. [CrossRef]

33. Douglas, W.R. Of pigs and men and research: A review of applications and analogies of the pig, sus scrofa, in human medical research. Space Life Sci. 1972, 3, 226-234. [CrossRef] [PubMed]

34. Lunney, J.K. Advances in swine biomedical model genomics. Int. J. Biol. Sci. 2007, 3, 179-184. [CrossRef] [PubMed]

35. Mahley, R.W.; Weisgraber, K.H.; Innerarity, T.; Brewer, H.B., Jr.; Assmann, G. Swine lipoproteins and atherosclerosis. Changes in the plasma lipoproteins and apoproteins induced by cholesterol feeding. Biochemistry 1975, 14, 2817-2823. [CrossRef] [PubMed]

36. Spurlock, M.E.; Gabler, N.K. The development of porcine models of obesity and the metabolic syndrome. J. Nutr. 2008, 138, 397-402. [PubMed]

37. Ashworth, C.J.; Finch, A.M.; Page, K.R.; Nwagwu, M.O.; McArdle, H.J. Causes and consequences of fetal growth retardation in pigs. Reprod. Suppl. 2001, 58, 233-246. [PubMed]

38. Foxcroft, G.R.; Dixon, W.T.; Novak, S.; Putman, C.T.; Town, S.C.; Vinsky, M.D. The biological basis for prenatal programming of postnatal performance in pigs. J. Anim. Sci. 2006, 84, E105-E112. [CrossRef] [PubMed]

39. Walsh Hentges, L.S.; Martin, R.J. Influence of genetic obesity on maternal and fetal serum and lipoprotein lipids in swine. Int. J. Obesity 1988, 12, 49-57.

40. Shelley, H.J.; Bassett, J.M.; Milner, R.D. Control of carbohydrate metabolism in the fetus and newborn. Br. Med. Bull. 1975, 31, 37-43. [CrossRef] [PubMed]

41. Hay, W.W., Jr. Recent observations on the regulation of fetal metabolism by glucose. J. Physiol. 2006, 572, 17-24. [CrossRef] [PubMed]

42. Rudolph, A.M. The fetal circulation and its response to stress. J. Dev. Physiol. 1984, 6, 11-19. [PubMed]

43. Haugen, G.; Hanson, M.; Kiserud, T.; Crozier, S.; Inskip, H.; Godfrey, K.M. Fetal liver-sparing cardiovascular adaptations linked to mother's slimness and diet. Circ. Res. 2005, 96, 12-14. [CrossRef] [PubMed] 
44. Jiang, B.; Godfrey, K.M.; Martyn, C.N.; Gale, C.R. Birth weight and cardiac structure in children. Pediatrics 2006, 117, e257-e261. [CrossRef] [PubMed]

45. Gentili, S.; Morrison, J.L.; McMillen, I.C. Intrauterine growth restriction and differential patterns of hepatic growth and expression of IGF1, PCK2 and HSDL1 mRNA in the sheep fetus in late gestation. Biol. Reprod. 2009, 80, 1121-1127. [CrossRef] [PubMed]

46. Latini, G.; De Mitri, B.; Del Vecchio, A.; Chitano, G.; De Felice, C.; Zetterström, R. Foetal growth of kidneys; liver and spleen in intrauterine growth restriction: "Programming" causing "metabolic syndrome" in adult age. Acta Paediatr. 2004, 93, 1635-1639. [CrossRef] [PubMed]

47. Coleman, R.A. Placental metabolism and transport of lipid. Fed. Proc. 1986, 45, 2519-2523. [PubMed]

48. Palinski, W. Maternal-fetal cholesterol transport in the placenta: Good, bad and target for modulation. Circ. Res. 2009, 104, 569-571. [CrossRef] [PubMed]

49. Woollett, L.A. The origins and roles of cholesterol and fatty acids in the fetus. Curr. Opin. Lipidol. 2001, 12, 305-312. [CrossRef] [PubMed]

50. Butte, N.F. Carbohydrate and lipid metabolism in pregnancy: Normal compared with gestational diabetes mellitus. Am. J. Clin. Nutr. 2000, 71, 1256S-1261S. [PubMed]

51. Wadsack, C.; Tabano, S.; Maier, A.; Hiden, U.; Alvino, G.; Cozzi, V.; Hüttinger, M.; Schneider, W.J.; Lang, U.; Cetin, I.; Desoye, G. Intrauterine growth restriction is associated with alterations in placental lipoprotein receptors and maternal lipoprotein composition. Am. J. Physiol. Endocrinol. Metabol. 2007, 292, E476-E484. [CrossRef] [PubMed]

52. Coleman, R.A.; Haynes, E.B. Microsomal and lysosomal enzymes of triacylglycerol metabolism in rat placenta. Biochem. J. 1984, 217, 391-397. [CrossRef] [PubMed]

53. Szabo, A.J.; De Lellis, R.; Grimaldi, R.D. Triglyceride synthesis by the human placenta. I. Incorporation of labeled palmitate into placental triglycerides. Am. J. Obstet. Gynecol. 1973, 115, 257-262. [CrossRef]

54. Vrijkotte, T.G.; Algera, S.J.; Brouwer, I.A.; van Eijsden, M.; Twickler, M.B. Maternal triglyceride levels during early pregnancy are associated with birth weight and postnatal growth. J. Pediatr. 2011, 159, 736-742. [CrossRef] [PubMed]

55. Bartels, A.; Egan, N.; Broadhurst, D.I.; Khashan, A.S.; Joyce, C.; Stapleton, M.; O’Mullane, J.; O’Donoghue, K. Maternal serum cholesterol levels are elevated from the 1st trimester of pregnancy: A cross-sectional study. J. Obstet. Gynaecol. 2012, 32, 747-752. [CrossRef] [PubMed]

56. Gonzalez-Bulnes, A.; Torres-Rovira, L.; Astiz, S.; Ovilo, C.; Sanchez-Sanchez, R.; Gomez-Fidalgo, E.; Perez-Solana, M.; Martin-Lluch, M.; Garcia-Contreras, C.; Vazquez-Gomez, M. Fetal Sex Modulates Developmental Response to Maternal Malnutrition. PLoS ONE 2015, 10, e0142158. [CrossRef] [PubMed]

57. Biezenski, J.J. Fetal lipid metabolism. Obstet. Gynecol. Ann. 1975, 4, 39-70.

58. Fain, J.; Scow, R. Fatty acid synthesis in vivo in maternal and fetal tissues in the rat. Am. J. Physiol. 1966, 210, $19-25$.

59. Hansen, A.E.; Wiese, H.F.; Adam, D.J.; Boelsche, A.N.; Haggard, M.E.; Davis, H.; Newsom, W.T.; Pesut, L. Influence of diet on blood serum lipids in pregnant women and newborn infants. Am. J. Clin. Nutr. 1964, 15, 11-19. [PubMed]

60. Johnston, P.V.; Johnson, O.C.; Kummerow, F.A. Non-transfer of trans fatty acids from mother to young. Proc. Soc. Exp. Biol. Med. 1957, 96, 760-762. [CrossRef] [PubMed]

61. Moore, C.E.; Dhopeshwarkar, G.A. Placental transport of trans fatty acids in the rat. Lipids 1980, 15, $1023-1028$. [CrossRef] [PubMed]

62. Pere, M.C. Materno-foetal exchanges and utilisation of nutrients by the foetus: Comparison between species. Reprod. Nutr. Dev. 2003, 43, 1-15. [CrossRef] [PubMed]

63. Larque, E.; Pagan, A.; Prieto, M.T.; Blanco, J.E.; Gil-Sanchez, A.; Zornoza-Moreno, M.; Ruiz-Palacios, M.; Gázquez, A.; Demmelmair, H.; Parrilla, J.J.; et al. Placental fatty acid transfer: A key factor in fetal growth. Ann. Nutr. Metab. 2014, 64, 247-253. [CrossRef] [PubMed]

64. Leskanich, C.O.; Noble, R.C. The comparative roles of polyunsaturated fatty acids in pig neonatal development. Br. J. Nutr. 1999, 81, 87-106. [PubMed]

65. Storlien, L.H.; Pan, D.A.; Kriketos, A.D.; O'Connor, J.; Caterson, I.D.; Cooney, G.J.; Jenkins, A.B.; Baur, L.A. Skeletal muscle membrane lipids and insulin resistance. Lipids 1996, 31, S261-S265. [CrossRef] [PubMed]

66. Li, Y.; Xu, S.; Zhang, X.; Yi, Z.; Cichello, S. Skeletal intramyocellular lipid metabolism and insulin resistance. Biophys. Rep. 2015, 1, 90-98. [CrossRef] [PubMed] 
67. Menon, N.K.; Moore, C.; Dhopeshwarkar, G.A. Effect of essential fatty acid deficiency on maternal, placental and fetal rat tissues. J. Nutr. 1981, 111, 1602-1610. [PubMed]

68. Attie, A.D.; Krauss, R.M.; Gray-Keller, M.P.; Brownlie, A.; Miyazaki, M.; Kastelein, J.J.; Lusis, A.J.; Stalenhoef, A.F.; Stoehr, J.P.; Hayden, M.R.; et al. Relationship between stearoyl-CoA desaturase activity and plasma triglycerides in human and mouse hypertriglyceridemia. J. Lipid Res. 2002, 43, 1899-1907. [CrossRef] [PubMed]

69. Hulver, M.W.; Berggren, J.R.; Carper, M.J.; Miyazaki, M.; Ntambi, J.M.; Hoffman, E.P.; Thyfault, J.P.; Stevens, R.; Dohm, G.L.; Houmard, J.A.; et al. Elevated stearoyl-CoA desaturase-1 expression in skeletal muscle contributes to abnormal fatty acid partitioning in obese humans. Cell Metab. 2005, 2, 251-261. [CrossRef] [PubMed]

70. Roden, M.; Price, T.B.; Perseghin, G.; Petersen, K.F.; Rothman, D.L.; Cline, G.W.; Shulman, G.I. Mechanism of free fatty acid-induced insulin resistance in humans. J. Clin. Invest. 1996, 97, 2859-2865. [CrossRef] [PubMed]

71. Poudyal, H.; Brown, L. Stearoyl-CoA desaturase: A vital checkpoint in the development and progression of obesity. Endocr. Metab. Immune Disord. Drug Targets 2011, 11, 217-231. [CrossRef] [PubMed]

72. Paillard, F.; Catheline, D.; Duff, F.L.; Bouriel, M.; Deugnier, Y.; Pouchard, M.; Daubert, J.C.; Legrand, P. Plasma palmitoleic acid; a product of stearoyl-coA desaturase activity; is an independent marker of triglyceridemia and abdominal adiposity. Nutr. Metab. Cardiovasc. Dis. 2008, 18, 436-440. [CrossRef] [PubMed]

73. Caron-Jobin, M.; Mauvoisin, D.; Michaud, A.; Veilleux, A.; Noel, S.; Fortier, M.P.; Julien, P.; Tchernof, A.; Mounier, C. Stearic acid content of abdominal adipose tissues in obese women. Nutr. Diabetes 2012, 2, e23. [CrossRef] [PubMed]

74. Leat, W.M. Studies on pig diets containing different amounts of linoleic acid. Br. J. Nutr. 1962, 16, 559-569. [CrossRef] [PubMed]

75. Adkisson, H.D.; Risener, F.S., Jr.; Zarrinkar, P.P.; Walla, M.D.; Christie, W.W.; Wuthier, R.E. Unique fatty acid composition of normal cartilage: Discovery of high levels of n-9 eicosatrienoic acid and low levels of n-6 polyunsaturated fatty acids. FASEB J. 1991, 5, 344-353. [PubMed]

76. Ovilo, C.; Fernandez, A.; Noguera, J.L.; Barragan, C.; Leton, R.; Rodríguez, C.; Mercadé, A.; Alves, E.; Folch, J.M.; Varona, L.; Toro, M. Fine mapping of porcine chromosome 6 QTL and LEPR effects on body composition in multiple generations of an Iberian by Landrace intercross. Genet. Res. 2005, 85, 57-67. [CrossRef] [PubMed]

77. British Society of Animal Science (BSAS). Nutrient Requirement Standards for Pigs; BSAS: Penicuik, UK, 2003.

78. Gonzalez-Bulnes, A.; Ovilo, C.; Lopez-Bote, C.J.; Astiz, S.; Ayuso, M.; Perez-Solana, M.L.; Sanchez-Sanchez, R.; Torres-Rovira, L. Gender-specific early postnatal catch-up growth after intrauterine growth retardation by food restriction in swine with obesity/leptin resistance. Reproduction 2012, 144, 269-278. [CrossRef] [PubMed]

79. Hausman, G.J.; Thomas, G.B. The development of perirenal fat depots in obese and lean pig fetuses. Int. J. Obes. 1987, 11, 545-557. [PubMed]

80. Walsh Hentges, L.S.; Martin, R.J. Serum and lipoprotein lipids of fetal pigs and their dams during gestation as compared with man. Biol. Neonate 1987, 52, 127-134. [CrossRef] [PubMed]

81. Vallet, J.L.; Freking, B.A. Differences in placental structure during gestation associated with large and small pig fetuses. J. Anim. Sci. 2007, 85, 3267-3275. [CrossRef] [PubMed]

82. Sutherland, M.R.; Gubhaju, L.; Black, M.J. Stereological assessment of renal development in a baboon model of preterm birth. Am. J. Nephrol. 2011, 33, 25-33. [CrossRef] [PubMed]

83. Ayres-Silva, J.P.; Manso, P.P.; Madeira, M.R.; Pelajo-Machado, M.; Lenzi, H.L. Sequential morphological characteristics of murine fetal liver hematopoietic microenvironment in Swiss Webster mice. Cell Tissue Res. 2011, 344, 455-469. [CrossRef] [PubMed]

84. Petersen, Y.M.; Burrin, D.G.; Sangild, P.T. GLP-2 has differential effects on small intestine growth and function in fetal and neonatal pigs. Am. J. Physiol. Regul. Integr. Comp. Physiol. 2001, 281, R1986-R1993. [PubMed]

85. Marmer, W.N.; Maxwell, R.J. Dry column method for the quantitative extraction and simultaneous class separation of lipids from muscle tissue. Lipids 1981, 16, 365-371. [CrossRef] [PubMed]

86. Segura, J.; Lopez-Bote, C.J. A laboratory efficient method for intramuscular fat analysis. Food Chem. 2014, 145, 821-825. [CrossRef] [PubMed] 
87. Lopez-Bote, C.J.; Rey, A.I.; Sanz, M.; Gray, J.I.; Buckley, D.J. Dietary vegetable oils and alpha-tocopherol reduce lipid oxidation in rabbit muscle. J. Nutr. 1997, 127, 1176-1182. [PubMed]

88. Olivares, A.; Rey, A.I.; Daza, A.; Lopez-Bote, C.J. High dietary vitamin A interferes with tissue alpha-tocopherol concentrations in fattening pigs: A study that examines administration and withdrawal times. Animal 2009, 3, 1264-1270. [CrossRef] [PubMed]

89. Ruiz, J.; Antequera, T.; Andres, A.; Petron, M.; Muriel, E. Improvement of a solid phase extraction method for analysis of lipid fractions in muscle foods. Anal. Chim. Acta 2004, 520, 201-205. [CrossRef]

90. Benzie, I.F.; Strain, J.J. The ferric reducing ability of plasma (FRAP) as a measure of "antioxidant power": The FRAP assay. Anal. Biochem. 1996, 239, 70-76. [CrossRef] [PubMed]

91. Larstad, M.; Ljungkvist, G.; Olin, A.C.; Toren, K. Determination of malondialdehyde in breath condensate by high-performance liquid chromatography with fluorescence detection. J. Chromatogr. B 2002, 766, 107-114. [CrossRef]

(c)

(C) 2017 by the authors. Licensee MDPI, Basel, Switzerland. This article is an open access article distributed under the terms and conditions of the Creative Commons Attribution (CC BY) license (http:/ / creativecommons.org/licenses/by/4.0/). 